\title{
Third-Order Asymptotics of Variable-Length Compression Allowing Errors
}

\author{
Yuta Sakai, Member, IEEE, Recep Can Yavas, Graduate Student Member, IEEE, and \\ Vincent Y. F. Tan, Senior Member, IEEE,
}

\begin{abstract}
This study investigates the fundamental limits of variable-length compression in which prefix-free constraints are not imposed (i.e., one-to-one codes are studied) and non-vanishing error probabilities are permitted. Due in part to a crucial relation between the variable-length and fixed-length compression problems, our analysis requires a careful and refined analysis of the fundamental limits of fixed-length compression in the setting where the error probabilities are allowed to approach either zero or one polynomially in the blocklength. To obtain the refinements, we employ tools from moderate deviations and strong large deviations. Finally, we provide the third-order asymptotics for the problem of variable-length compression with non-vanishing error probabilities. We show that unlike several other informationtheoretic problems in which the third-order asymptotics are known, for the problem of interest here, the third-order term depends on the permissible error probability.
\end{abstract}

Index Terms-Variable-length compression, Third-order asymptotics, Average codeword lengths, Moderate deviations, Cramér-type large deviations, Strong large deviations

\section{INTRODUCTION}

Characterizing fundamental limits of coding problems is the central goal in information theory. The class of variablelength compression problems (i.e., fixed-to-variable length coding problems) constitute a classical and important family of information-theoretic problems in view of their multitude of practical applications. Han [1] considered the problem of variable-length compression with prefix-free constraints allowing a small (i.e., vanishing) error probability. He then derived the first-order optimal coding rate for a general source when the error probability is required to vanish with increasing blocklengths. Later, Koga and Yamamoto [2] derived the firstorder optimal coding rate in the regime of non-vanishing error probabilities. In the particular case of a stationary memoryless source $X$, their work [2] showed that

$$
L_{\text {prefix }}^{*}\left(\varepsilon \mid X^{n}\right)=n(1-\varepsilon) H(X)+\mathrm{o}(n)
$$

as $n \rightarrow \infty$ for fixed $0 \leq \varepsilon \leq 1$, where $L_{\text {prefix }}\left(\varepsilon \mid X^{n}\right)$ denotes the minimum of average codeword lengths of binary prefixfree codes for $n$ i.i.d. copies $X^{n}$ of $X$ in which the error

This work is supported by a Singapore National Research Foundation (NRF) Fellowship (R-263-000-D02-281). This work was presented in part at the International Symposium on Information Theory and Its Applications (ISITA) in Kapolei, Hawaii, USA in October 2020.

Y. Sakai is with the Department of Electronics and Computer Science, Graduate School of Engineering, University of Hyogo, Email: yuta.sakai@eng.u-hyogo.ac.jp. R. C. Yavas is with the Department of Electrical Engineering, California Institute of Technology, Email ryavas@caltech.edu. V. Y. F. Tan is with the Department of Electrical and Computer Engineering and the Department of Mathematics, National University of Singapore, Singapore, Email: vtan@nus.edu.sg. probability is at most $\varepsilon$, and $H(X)$ stands for the entropy of $X$ measured in bits. Hence, in general, the strong converse property (cf. [3]) fails to hold in variable-length compression problems. The equality in (1) also demonstrates the utility in using both variable-length coding as well as permitting nonvanishing error probabilities. This is because the first-order fundamental limit is reduced to $(1-\varepsilon) H(X)$ if we permit an error probability $\varepsilon>0$. If one were to use fixed-length codes or demand that the error probabilities are vanishing, one cannot compress a source $X$ with entropy $H(X)$ with rate strictly below $H(X)$. This formalism is an alternative to lossy source coding (rate-distortion) for the purpose of reducing the compression rate. In lossy compression, the source suffers from some distortion; here the source sequence is either reproduced perfectly or an arbitrary sequence is generated (with probability $\varepsilon$ ).

In this paper, we consider variable-length compression problems without prefix-free constraints. In the zero-error setting, this class of fixed-to-variable length codes is often known as one-to-one codes. While the redundancy ${ }^{1}$ of a prefix-free code is always nonnegative, the redundancy of a one-to-one code can be negative (cf. [4], [5]). ${ }^{2}$ In fact, Szpankowski and Verdú [7] proved an asymptotic expansion of the smallest redundancies of one-to-one codes for a stationary memoryless source $X$. They showed that for finitely supported non-equiprobable $X$,

$$
L^{*}\left(0 \mid X^{n}\right)=n H(X)-\frac{1}{2} \log n+\mathrm{O}(1)
$$

as $n \rightarrow \infty$, where $L^{*}\left(0 \mid X^{n}\right)$ denotes the minimum of average codeword lengths of one-to-one codes for $X^{n}$. Furthermore, Szpankowski [6] refined the remainder term $+\mathrm{O}(1)$ in (2) when $X$ is a Bernoulli source, and clarified necessary and sufficient conditions on $X$ for which the dominant term within the $+\mathrm{O}(1)$ remainder term converges or oscillates. On the other hand, in the regime of non-vanishing error probabilities, Kostina, Polyanskiy, and Verdú [8] derived the second-order optimal coding rate of this fundamental limit for a stationary memoryless source. They [8] showed that

$$
L^{*}\left(\varepsilon \mid X^{n}\right)=n(1-\varepsilon) H(X)-\sqrt{\frac{n V(X)}{2 \pi}} \mathrm{e}^{-\Phi^{-1}(\varepsilon)^{2} / 2}+\mathrm{O}(\log n)
$$

\footnotetext{
${ }^{1}$ The redundancy of a fixed-to-variable length code is defined as "its average codeword length minus the entropy of the source."

${ }^{2}$ Hence, the redundancy of a one-to-one code is sometimes termed as the anti-redundancy (cf. [6]).
} 
as $n \rightarrow \infty$ for fixed $0 \leq \varepsilon \leq 1$, provided that the variance and the absolute third central moment of the information density $-\log P_{X}(X)$ are positive and finite, respectively, where $L^{*}\left(\varepsilon \mid X^{n}\right)$ stands for the minimum of average codeword lengths of non-prefix-free codes for $X^{n}$ in which the error probability is at most $\varepsilon$, the quantity $V(X)$ stands for the varentropy of $X$ measured in bits squared per source symbol (cf. [9]), and $\Phi^{-1}(\cdot)$ stands for the inverse of the Gaussian cumulative distribution function. The intuition for the somewhat unusual dispersion term in (3) can be found in $[8$, Section II.E]. It is clear that (3) is consistent with (2) when $\varepsilon=0$ because $\Phi^{-1}(0)=-\infty$ and so the second-order term vanishes when $\varepsilon=0$.

\section{A. Contributions of This Study}

In this study, we consider refinements of (2) and (3) simultaneously. In particular, we generalize Szpankowski and Verdú's work [7] from the zero-error setting (i.e., $\varepsilon=0$ ) to the setting with non-vanishing error probabilities (i.e., $\varepsilon>0$ ). More importantly, we refine the $+\mathrm{O}(\log n)$ remainder term in Kostina et al.'s second-order asymptotic result [8]. We show that this term equals $-((1-\varepsilon) \log n) / 2$. To do so, we use a crucial relation between variable-length and fixedlength codes and their fundamental limits (cf. [10]). We derive higher-order asymptotics of the variable-length compression problem by leveraging that of the fixed-length compression problem. In this strategy, we have to consider the fixed-length compression problem in which error probability approaches zero or one polynomially in the blocklength. To deal with these sequences of error probabilities that tend to the boundary of the open interval $(0,1)$, we apply techniques from moderate deviations and strong large deviations (cf. [11]-[13]). The resulting higher-order asymptotics of the fixed-length compression problem yields our desired third-order asymptotic expansion of the fundamental limit of the variable-length compression problem. Somewhat interestingly, unlike several other information-theoretic problems in which the third-order asymptotics are known, for the problem of interest here, the third-order term depends on the permissible error probability $\varepsilon$. Finally, we believe that the new mathematical results derived here (cf. Lemmas 7 and 9) may be of independent interest in information theory and beyond.

\section{B. Related Works}

1) Higher-Order Asymptotics of Fixed-Length Compression: In view of the recent developments of the second- and thirdorder asymptotics of coding problems [14]-[20], given a source $X$ with a countable source alphabet $X$, it is well-known that

$$
\log M^{*}(n, \varepsilon)=n H(X)-\sqrt{n V(X)} \Phi^{-1}(\varepsilon)-\frac{1}{2} \log n+\mathrm{O}(1)
$$

as $n \rightarrow \infty$ for fixed $0<\varepsilon<1$, provided that the variance and the absolute third central moment of the information density $-\log P_{X}(X)$ are positive and finite, respectively, where $\log$ denotes the logarithm to the base- 2 and $M^{*}(n, \varepsilon)$ stands for the smallest cardinality a set $\mathcal{A} \subset \mathcal{X}^{n}$ in which the $P_{X^{n-}}$ probability of $\mathcal{A}$ is at least $1-\varepsilon$. In his seminal work, Strassen [14] derived the fourth-order asymptotics of the fixed-length compression with non-vanishing error probabilities. Strassen derived this by considering the Edgeworth expansion, a higherorder asymptotic expansion that goes beyond the central limit theorem (cf. [11], [12]). In channel coding problems, Moulin [19] established certain bounds on the fourth-order optimal coding rate under some regularity conditions on discrete memoryless channels. Recently, Hayashi [20] investigated the fourth-order asymptotics of various information-theoretic problems.

2) Moderate Deviations Analysis: In information theory, there are two main types of coding theorems that provide refinements to capacity results, theorems concerning error exponents and second-order asymptotics. The former evaluates the exponential decay of error probabilities when coding rates are fixed; the latter evaluates the deviations from the firstorder fundamental limits (which are typically of order $1 / \sqrt{n}$ ) when error probabilities are fixed. The moderate deviations analysis of coding problems lie in between these two asymptotic regimes. Moderate deviations examines the interplay between the sub-exponential decay of error probabilities and the deviation from the first-order fundamental limits which are typically of order $\kappa_{n} / \sqrt{n}$ where the positive sequence $\kappa_{n}=\omega(1) \cap \mathrm{o}(\sqrt{n})$ as $n \rightarrow \infty$. See [21, Section I] for earlier works on moderate deviations in information theory. Most notably, in channel coding, Altuğ and Wagner [21] investigated the sub-exponential rate of decay of the error probabilities when the coding rate approaches the capacity slower than that in the study of the second-order asymptotics [14]-[18], [20]. Some of these techniques will turn out to be useful for the solution of our problem.

3) Exact Asymptotics of Error Probabilities: The study of strong large deviations [13, Theorem 3.7.4] and [12, Chapter VIII.4], or exact asymptotics, is a refinement of the large deviations principle. While the rate function in the large deviations principle characterizes the exponential decay of the complementary cumulative distribution function of a sum of independent r.v.'s, the theorems in the study of strong large deviations further characterize its sub-exponential decay, and such sub-exponential terms are often referred to as pre-factors. The classical error exponent analysis of channel coding theorems has been refined in the context of the exact asymptotics of the error probability (cf. [22]-[26]). Again, exact asymptotics will play a crucial role in the estimates of some rates and error probabilities in our work.

4) Refined Asymptotics of Variable-Length Compression with the Excess Length Constraint: Instead of the average codeword length, Merhav [27] introduced another performance criterion, namely, the excess length. Under this setting, one is interested in finding a threshold under which the complementary cumulative distribution function of the codeword length evaluated at this threshold is suitably upper bounded. This excess length constraint is closely related to the fixed-length compression problem (cf. [9]). Third-order asymptotic expansions under the excess length constraint of type size codes for universal variable-length compression were investigated 
by Kosut and Sankar [28]. Iri and Kosut [29] generalized this work by considering parametric sources defined by finitedimensional exponential families. Finally, Nomura and Yagi [30] established general formulas for the first- and secondorder terms of the fundamental limits of this class of problems for a general source.

\section{Paper Organization}

The rest of this paper is organized as follows: Section II introduces basic definitions and notations in this study. Section III revisits previous works summarized in (2) and (3), and states our main result as their integration. Section IV proves our main result by presenting several technical lemmas. Section V investigates moderate deviations and strong large deviations analyses for the fixed-length compression. Section VI concludes this study.

\section{Preliminaries}

\section{A. Random Variables and Discrete Memoryless Sources}

In this subsection, we introduce basic notions in probability theory, a discrete memoryless source and its information measures. Let $(\Omega, \mathcal{F}, \mathbb{P})$ the underlying probability space, and $Z$ a real-valued r.v. Denote by $P_{Z}:=\mathbb{P} \circ Z$ the probability distribution induced by $Z$. We say that $Z$ is a lattice r.v. if it is discrete and there exists a positive constant $d$ such that $\mathcal{D}(Z):=\left\{z_{1}-z_{2} \mid P_{Z}\left(z_{1}\right) P_{Z}\left(z_{2}\right)>0\right\}$ is a subset of $d \mathbb{Z}:=\{\ldots,-2 d,-d, 0, d, 2 d, \ldots\}$. Otherwise, we say that $Z$ is a nonlattice r.v. For a lattice r.v. $Z$, its maximal span is defined by the maximum of positive constants $d$ satisfying $\mathcal{D}(Z) \subset d \mathbb{Z}$.

Given a real-valued r.v. $Z$ and a real number $0<\varepsilon<1$, define the $\varepsilon$-cutoff transformation action of $Z$ [8, Equation (13)] by

$$
\langle Z\rangle_{\varepsilon}:= \begin{cases}Z & \text { if } Z<\eta, \\ B Z & \text { if } Z=\eta, \\ 0 & \text { if } Z>\eta,\end{cases}
$$

where $B$ is the Bernoulli r.v. with parameter $1-\beta$ in which $B$ is independent of $Z$, and two real parameters $\eta \in \mathbb{R}$ and $0 \leq \beta<1$ are chosen so that

$$
\mathbb{P}\{Z>\eta\}+\beta \mathbb{P}\{Z=\eta\}=\varepsilon .
$$

Consider a countably infinite alphabet $\mathcal{X}$ and an $\mathcal{X}$-valued r.v. $X$. In this study, i.i.d. copies $\left\{X_{i}\right\}_{i=1}^{\infty}$ of $X$ play the role of a discrete memoryless source, and we simply call $X$ the source. A source $X$ is said to be finitely supported if the support $\operatorname{supp}(X):=\left\{x \in \mathcal{X} \mid P_{X}(x)>0\right\}$ is finite. We say that $X$ is a lattice source if $\log P_{X}(X)$ is a lattice r.v., where $\log$ stands for the logarithm to the base-2. On the other hand, we say that $X$ is a nonlattice source if $\log P_{X}(X)$ is a nonlattice r.v. For a lattice source $X$, denote by $d_{X}$ the maximal span of $\log P_{X}(X)$. For convenience, we set $d_{X}$ to be zero if $X$ is a nonlattice source.

Define the Shannon entropy, the Rényi entropy, the varentropy, and one-sixth of the skewness of $X$ as

$$
H(X):=\sum_{x \in \operatorname{supp}(X)} P_{X}(x) \log \frac{1}{P_{X}(x)},
$$

$$
\begin{aligned}
H_{\alpha}(X) & :=\frac{1}{1-\alpha} \log \left(\sum_{x \in \operatorname{supp}(X)} P_{X}(x)^{\alpha}\right), \\
V(X) & :=\sum_{x \in \operatorname{supp}(X)} P_{X}(x)\left(\log \frac{1}{P_{X}(x)}-H(X)\right)^{2}, \\
S(X) & :=\frac{1}{6} \sum_{x \in \operatorname{supp}(X)} P_{X}(x)\left(\frac{-\log P_{X}(x)-H(X)}{\sqrt{V(X)}}\right)^{3},
\end{aligned}
$$

respectively. Throughout this study, assume that $V(X)>0$, i.e., assume that $X$ is not uniformly distributed on a finite subalphabet $\mathcal{A} \subset \mathcal{X}$.

Similar to a notion in probability theory (cf. [12, Chapter VIII.2]), we define the following condition on a source $X$.

Definition 1. We say that a source $X$ satisfies Cramér's condition if $H_{\alpha}(X)$ is finite for some $0<\alpha<1$.

Remark 1. The Rényi entropy $H_{\alpha}(X)$ can be thought of as a monotone function of the cumulant generating function of the information density $-\log P_{X}(X)$, i.e., we readily see that

$$
(1-\alpha) H_{\alpha}(X)=\log \mathbb{E}\left[2^{(1-\alpha) \log P_{X}(X)}\right] .
$$

Namely, Cramér's condition on $X$ ensures the existence of the $k$-th moment $\mathbb{E}\left[\log ^{k} P_{X}(X)\right]$ for every $k \geq 1$, i.e., the quantities $H(X), V(X)$, and $S(X)$ are finite in this case. Note that there exists a source $X$ such that $H(X), V(X)$, and $S(X)$ are finite but Cramér's condition fails to hold (see, e.g., [31, Example 5]). On the other hand, since $H_{\alpha}(X) \leq \log |\operatorname{supp}(X)|$ for every $\alpha \geq 0$, it is easy to see that every finitely supported source $X$ satisfies Cramér's condition.

\section{B. Gaussian Distributions}

Define the Gaussian probability density function and the Gaussian cumulative distribution function as

$$
\varphi(u):=\frac{1}{\sqrt{2 \pi}} \mathrm{e}^{-u^{2} / 2}, \quad \text { and } \quad \Phi(u):=\int_{-\infty}^{u} \varphi(t) \mathrm{d} t
$$

for $u \in \mathbb{R}$, respectively. Sometimes, we will also find it convenient to use $\mathrm{Q}(u):=1-\Phi(u)$, the Gaussian complementary cumulative distribution function. Moreover, define

$$
\begin{aligned}
f_{\mathrm{G}}(s) & := \begin{cases}\varphi\left(\Phi^{-1}(s)\right) & \text { if } 0<s<1, \\
0 & \text { if } s=0 \text { or } s=1,\end{cases} \\
g_{\mathrm{G}}(s) & := \begin{cases}f_{\mathrm{G}}(\varepsilon) \Phi^{-1}(\varepsilon) & \text { if } 0<s<1, \\
0 & \text { if } s=0 \text { or } s=1,\end{cases}
\end{aligned}
$$

for $0 \leq s \leq 1$, where $\Phi^{-1}(\cdot)$ denotes the inverse function of $\Phi(\cdot)$. It is known that

$$
\begin{gathered}
\Phi^{-1}(s) \sim-\sqrt{2 \ln \frac{1}{s}}, \\
f_{\mathrm{G}}(s) \sim s \sqrt{2 \ln \frac{1}{s}}
\end{gathered}
$$

as $s \rightarrow 0^{+}$(cf. [32, Lemma 5.2]), where ln stands for the natural logarithm. Thus, we see that

$$
\lim _{s \rightarrow 0^{+}} f_{\mathrm{G}}(\varepsilon) \Phi^{-1}(\varepsilon)=-\lim _{s \rightarrow 1^{-}} f_{\mathrm{G}}(\varepsilon) \Phi^{-1}(\varepsilon)=\lim _{s \rightarrow 0^{+}} 2 s \ln s=0,
$$


implying that the definitions of $g_{\mathrm{G}}(s)$ at $s=0$ and at $s=1$ are consistent with the limits as $s \rightarrow 0^{+}$and as $s \rightarrow 1^{-}$, respectively. The following lemma shows a higherorder asymptotic expansion of $\Phi^{-1}(\cdot)$ beyond that presented in (15).

Lemma 1 ([33]). It holds that

$$
\Phi^{-1}(s)^{2}=2 \ln \frac{1}{2 \sqrt{\pi} s}-\ln \ln \frac{1}{2 \sqrt{\pi} s}+\mathrm{O}\left(\frac{\ln \ln (1 / s)}{\ln (1 / s)}\right)
$$

as $s \rightarrow 0^{+}$.

The following lemma is employed to integrate polynomials of $\Phi^{-1}(\cdot)$.

Lemma 2. Given $0 \leq a<b \leq 1$, it holds that

$$
\begin{aligned}
& \int_{a}^{b} \Phi^{-1}(s) \mathrm{d} s=f_{\mathrm{G}}(a)-f_{\mathrm{G}}(b), \\
& \int_{a}^{b} \Phi^{-1}(s)^{2} \mathrm{~d} s=(b-a)-g_{\mathrm{G}}(b)+g_{\mathrm{G}}(a),
\end{aligned}
$$

Proof of Lemma 2: Elementary calculations yield these formulas, and we omit the proof details here.

\section{Asymptotic Notations}

In this paper, we use the following asymptotic notations to express our asymptotic expansions in source coding problems. Let $\mathcal{I}_{n}$ be a sequence of real intervals, and $\mathcal{I}=\bigcup_{n} \mathcal{I}_{n}$. Consider two sequences $\left\{f_{n}\right\}_{n=1}^{\infty}$ and $\left\{g_{n}\right\}_{n=1}^{\infty}$ of real-valued functions on $\mathcal{I}$, and a sequence $\left\{a_{n}\right\}_{n=1}^{\infty}$ of positive numbers. For fixed $t \in \mathcal{I}$, we say that $f_{n}(t)=g_{n}(t)+\mathrm{O}\left(a_{n}\right)$ as $n \rightarrow \infty$ if

$$
\limsup _{n \rightarrow \infty} \frac{\left|f_{n}(t)-g_{n}(t)\right|}{a_{n}}<\infty
$$

and that $f_{n}(t)=g_{n}(t)+\mathrm{o}\left(a_{n}\right)$ as $n \rightarrow \infty$ if

$$
\lim _{n \rightarrow \infty} \frac{\left|f_{n}(t)-g_{n}(t)\right|}{a_{n}}=0 .
$$

In particular, we say that $f_{n}(t)=g_{n}(t)+\mathrm{O}\left(a_{n}\right)$ uniformly on $\mathcal{I}_{n}$ as $n \rightarrow \infty$ if

$$
\limsup _{n \rightarrow \infty} \frac{1}{a_{n}} \sup _{t \in \mathcal{I}_{n}}\left|f_{n}(t)-g_{n}(t)\right|<\infty
$$

and that $f_{n}(t)=g_{n}(t)+\mathrm{o}\left(a_{n}\right)$ uniformly on $\mathcal{I}_{n}$ as $n \rightarrow \infty$ if

$$
\lim _{n \rightarrow \infty} \frac{1}{a_{n}} \sup _{t \in \mathcal{I}_{n}}\left|f_{n}(t)-g_{n}(t)\right|=0 .
$$

In this study, these uniform convergence properties on a sequence of intervals are used in the moderate deviations analysis to investigate higher-order asymptotics of the fixedlength compression problem in which the error probabilities are asymptotically close to zero or one for sufficiently large codeword lengths; see Section IV-A for details.

\section{VARIABLE-LENGTH COMPRESSION}

A. Variable-Length Compression Allowing Errors-Revisited

In this subsection, we revisit the previous results stated in (2) and (3) formally. Consider compressing a discrete memoryless source $X$ into a finite-length binary string. Let

$$
\{0,1\}^{*}:=\{\varnothing\} \cup\left(\bigcup_{n=1}^{\infty}\{0,1\}^{n}\right)
$$

be the set of finite-length binary strings containing the empty string $\varnothing$. Denote by $\ell:\{0,1\}^{*} \rightarrow \mathbb{N} \cup\{0\}$ the length function of a binary string, i.e., $\ell(\varnothing)=0, \ell(0)=\ell(1)=1, \ell(00)=$ $\ell(01)=\ell(10)=\ell(11)=2$, etc.

Definition 2. An $(L, \varepsilon)$-code for a source $X$ is a pair of a stochastic encoder $F: \mathcal{X} \rightarrow\{0,1\}^{*}$ and a stochastic decoder $G:\{0,1\}^{*} \rightarrow \mathcal{X}$ such that

$$
\begin{array}{r}
\mathbb{E}[\ell(F(X))] \leq L, \\
\mathbb{P}\{X \neq G(F(X))\} \leq \varepsilon .
\end{array}
$$

Given a permissible probability of error $0 \leq \varepsilon \leq 1$, denote by $L^{*}(\varepsilon \mid X)$ the infimum of $L>0$ such that an $(L, \varepsilon)$-code exists for $X$. We recall the definition of $\langle\cdot\rangle_{\varepsilon}$ in (5). It is known (cf. [8, Equation (26)] and [34, Lemmas 1 and 5]) that

$$
L^{*}\left(\varepsilon \mid X^{n}\right)=\mathbb{E}\left[\left\langle\left\lfloor\log \varsigma_{n}^{-1}\left(X^{n}\right)\right\rfloor\right\rangle_{\varepsilon}\right],
$$

where $\varsigma_{n}:\{1,2,3, \ldots\} \rightarrow \mathcal{X}^{n}$ is an arbitrary bijection satisfying $^{3}$

$$
P_{X^{n}}\left(\varsigma_{n}(1)\right) \geq P_{X^{n}}\left(\varsigma_{n}(2)\right) \geq P_{X^{n}}\left(\varsigma_{n}(3)\right) \geq \cdots,
$$

and $\lfloor\cdot\rfloor:=\max \{z \in \mathbb{Z} \mid z \leq \cdot\}$ denotes the floor function. Since, in the following, we will mostly be working with the inverse $\varsigma_{n}^{-1}$, for notational conciseness, we write this as $\gamma_{n}$, i.e.,

$$
\gamma_{n}\left(x^{n}\right):=\varsigma_{n}^{-1}\left(x^{n}\right) \text { for all } x^{n} \in \mathcal{X}^{n} .
$$

Note that the right-hand side of (28) is not single-letterized, and we are interested to determine asymptotic expansions of $L^{*}\left(\varepsilon \mid X^{n}\right)$ as $n \rightarrow \infty$ in a computable form.

The following theorem is a known second-order asymptotic result for this problem.

Theorem 1 (Kostina, Polyanskiy, and Verdú [8, Theorem 4]). Given a fixed $0 \leq \varepsilon \leq 1$ and a source $X$, it holds that

$$
L^{*}\left(\varepsilon \mid X^{n}\right)=n(1-\varepsilon) H(X)-\sqrt{n V(X)} f_{\mathrm{G}}(\varepsilon)+\mathrm{O}(\log n)
$$

as $n \rightarrow \infty$, provided that $\mathbb{E}\left[\log ^{3} P_{X}(X)\right]$ is finite.

In [8], Theorem 1 was proven by establishing the one-shot bounds ${ }^{4}$

$$
\begin{gathered}
\mathbb{E}\left[\left\langle\log \frac{1}{P_{X^{n}}\left(X^{n}\right)}\right\rangle_{\varepsilon}\right]-\log (1+n H(X))-\log \mathrm{e} \\
\leq L^{*}\left(\varepsilon \mid X^{n}\right) \leq \mathbb{E}\left[\left\langle\log \frac{1}{P_{X^{n}}\left(X^{n}\right)}\right\rangle_{\varepsilon}\right]
\end{gathered}
$$

\footnotetext{
${ }^{3}$ Namely, the bijection $\boldsymbol{S}_{n}$ plays the role of a decreasing rearrangement of $P_{X^{n}}(\cdot)$.

${ }^{4}$ When $\varepsilon=0$, the lower bound specializes Alon and Orlitsky's bound [5], and the upper bound specializes Wyner's bound [4].
} 
and the asymptotic expansion ${ }^{5}$

$$
\mathbb{E}\left[\left\langle\log \frac{1}{P_{X^{n}}\left(X^{n}\right)}\right\rangle_{\varepsilon}\right]=n(1-\varepsilon) H(X)-\sqrt{n V(X)} f_{\mathrm{G}}(\varepsilon)+\mathrm{O}(1)
$$

as $n \rightarrow \infty$. Roughly speaking, this proof strategy converts the analysis of $\log \gamma_{n}\left(X^{n}\right)$ to that of the information density $-\log P_{X^{n}}\left(X^{n}\right)$; see (28).

For an asymptotic relation (in an almost sure sense) between $\log \gamma_{n}\left(X^{n}\right)$ and $-\log P_{X^{n}}\left(X^{n}\right)$ up to the $+\mathrm{o}\left(\kappa_{n} \log n\right)$ term with any slowly divergent positive sequence $\left\{\kappa_{n}\right\}_{n=1}^{\infty}$, we refer the reader to the study of pointwise redundancy studied by Kontoyiannis and Verdú [9, Section IV].

In the particular case of $\varepsilon=0$ and finitely supported $X$, Theorem 1 can be refined as follows:

Theorem 2 (Szpankowski and Verdú [7, Theorem 4]). For a finitely supported source $X$, it holds that

$$
L^{*}\left(0 \mid X^{n}\right)=n H(X)-\frac{1}{2} \log n+\mathrm{O}(1)
$$

as $n \rightarrow \infty$.

In [7], Theorem 2 was proven via complex analysis socalled the analytic Poissonization and de-Poissonization (cf. [35]) and Stirling's formula to approximate multinomial coefficients.

\section{B. Main Result-Higher-Order Asymptotics of Variable- Length Compression}

The following theorem constitutes the main result of the paper.

Theorem 3. Let $0<\varepsilon \leq 1$ be fixed. If the source $X$ satisfies Cramér's condition, then

$$
\begin{aligned}
& L^{*}\left(\varepsilon \mid X^{n}\right) \\
& =n(1-\varepsilon) H(X)-\sqrt{n V(X)} f_{\mathrm{G}}(\varepsilon)-\frac{1-\varepsilon}{2} \log n+\mathrm{O}(1)
\end{aligned}
$$

as $n \rightarrow \infty$. On the other hand, if $\varepsilon=0$ and $X$ is finitely supported, then (34) holds.

Our main result in (35) provides a third-order refinement of Kostina, Polyanskiy, and Verdú's second-order result in (3) for variable-length lossless compression with errors [8, Theorem 4].

We prove Theorem 3 in the next section.

The proof outline of Theorem 3 is as follows: Since every codeword length is a nonnegative integer, it is known that

$$
L^{*}\left(0 \mid X^{n}\right)=\sum_{k=1}^{\infty} \mathbb{P}\left\{\log \gamma_{n}\left(X^{n}\right) \geq k\right\}
$$

(cf. [9, Section III]). Given $0<\varepsilon \leq 1$, this identity can be readily extended as

$$
L^{*}\left(\varepsilon \mid X^{n}\right)=\sum_{k=1}^{\tilde{\xi}_{n}} \mathbb{P}\left\{\log \gamma_{n}\left(X^{n}\right) \geq k\right\}-\varepsilon \tilde{\xi}_{n},
$$

\footnotetext{
${ }^{5}$ This asymptotic expansion was proven by Berry-Esseen-type bounds (cf [11, Chapter XVI.5] and [12, Chapter V.4]).
}

where the integer $\tilde{\xi}_{n}=\tilde{\xi}_{n}(\varepsilon, X)$ is chosen so that

$$
\begin{aligned}
& \mathbb{P}\left\{\log \gamma_{n}\left(X^{n}\right) \geq \tilde{\xi}_{n}\right\} \geq \varepsilon, \\
& \mathbb{P}\left\{\log \gamma_{n}\left(X^{n}\right)>\tilde{\xi}_{n}\right\}<\varepsilon .
\end{aligned}
$$

Here, the complementary cumulative distribution function $\mathbb{P}\left\{\log \gamma_{n}\left(X^{n}\right)>k\right\}$ corresponds to the overflow probability of codeword lengths (cf. [9], [27]-[30], [36]), and can be thought of as the minimum average probability of error for $n$-to- $k$ binary block codes for the source $X^{n}$. Namely, the average codeword length $L^{*}\left(\varepsilon \mid X^{n}\right)$ of variable-length compression can be analyzed via the fundamental limits of fixed-length compression via its relation to $\log \gamma_{n}\left(X^{n}\right)$ as stated in (28). ${ }^{6}$ In particular in Lemma 4, we show that the expectation of the $\varepsilon$-cutoff transformation action on $\log \gamma_{n}\left(X^{n}\right)$ (with the floor operator removed) is related to the fixed-length source coding asymptotics $M^{*}(n, s)$ as follows:

$$
\mathbb{E}\left[\left\langle\log \gamma_{n}\left(X^{n}\right)\right\rangle_{\varepsilon}\right] \approx \int_{\varepsilon}^{1} \log M^{*}(n, s) \mathrm{d} s .
$$

To bound the integral on the right-hand-side, we split it into two parts (as error probabilities close to 0 or 1 are difficult to deal with using central limit-type techniques), namely

$$
\begin{aligned}
& \int_{\varepsilon}^{1} \log M^{*}(n, s) \mathrm{d} s \\
& =\underbrace{\int_{\varepsilon}^{1-n^{-1}} \log M^{*}(n, s) \mathrm{d} s}_{=: \mathrm{A}}+\underbrace{\int_{1-n^{-1}}^{1} \log M^{*}(n, s) \mathrm{d} s}_{=: \mathrm{B}} .
\end{aligned}
$$

Integral B only contributes an $\mathrm{O}(1)$ term. Estimating integral $\mathrm{A}$ is the essence of the whole proof. It requires us to estimate $\log M^{*}\left(n, \varepsilon_{n}\right)$ for error probabilities $\varepsilon_{n}$ that are vanishing or growing polynomially fast, e.g., $\varepsilon_{n} \approx n^{-1}$ or $\varepsilon_{n} \approx 1-n^{-1}$. Thus, one of our main endeavors and contributions (done in Lemma 7) is to estimate $\log M^{*}\left(n, 1 / n^{r}\right)$ and $\log M^{*}\left(n, 1-1 / n^{r}\right)$ for $r>0$, i.e., the fundamental limits of fixed-length source coding when the error probability or success probability is polynomially small. This requires techniques from the theory of moderate deviations and strong large deviations.

\section{Proof of Theorem 3}

In this section, we prove Theorem 3 by presenting some technical lemmas.

\section{A. Moderate Deviations and Strong Large Deviations of Fixed- Length Compression}

Before investigating higher-order asymptotic expansions of the variable-length compression problem, we now consider the fixed-length compression problem for a stationary memoryless source $X^{n}$. An $(n, M, \varepsilon)$-code for the source $X$ consists of an encoder $f: X^{n} \rightarrow\{1,2, \ldots, M\}$ and a decoder $g:\{1,2, \ldots, M\} \rightarrow \mathcal{X}^{n}$ such that

$$
\mathbb{P}\left\{X^{n} \neq g\left(f\left(X^{n}\right)\right)\right\} \leq \varepsilon .
$$

\footnotetext{
${ }^{6}$ This relation was mentioned by S. Verdú in his Shannon Lecture [10].
} 
Denote by $M^{*}(n, \varepsilon)$ the minimum of $M \in \mathbb{N}$ such that an $(n, M, \varepsilon)$-code exists for the source $X$. In other words, it is defined as

$$
M^{*}(n, \varepsilon)=\min _{\substack{\mathcal{A} \subset \mathcal{X}: \\ P_{X^{n}}(\mathcal{A}) \geq 1-\varepsilon}}|\mathcal{A}| .
$$

The following lemma is a result of judiciously combining the use of a moderate deviations theorem and a strong large deviations theorem [11]-[13].

Lemma 3. Suppose that $X$ satisfies Cramér's condition stated in Definition 1. Let $\left\{\varepsilon_{n}\right\}_{n=1}^{\infty}$ be a real sequence on $(0,1)$. If

$$
\frac{1}{n^{r}} \leq \varepsilon_{n} \leq 1-\frac{1}{n^{r}}
$$

for some positive real $r$ and for sufficiently large $n$. Then ${ }^{7}$

$$
\begin{array}{rl}
\log M^{*}\left(n, \varepsilon_{n}\right)=n & H(X)-\sqrt{n V(X)} \Phi^{-1}\left(\varepsilon_{n}\right)-\frac{1}{2} \log n \\
& +\left(S(X)-\frac{\log \mathrm{e}}{2}\right) \Phi^{-1}\left(\varepsilon_{n}\right)^{2}+\mathrm{O}(1)
\end{array}
$$

as $n \rightarrow \infty$, where $S(X)$ is defined in (10).

Proof of Lemma 3: See Section V-C.

Remark 2 (Refinements to the source dispersion term in the moderate deviations regime). Define

$$
D^{*}(n, \varepsilon):=\frac{\log M^{*}(n, \varepsilon)-n H(X)}{\sqrt{n V(X)}} .
$$

It is well-known that $D^{*}(n, \varepsilon) \rightarrow \Phi^{-1}(1-\varepsilon)$ as $n \rightarrow \infty$ for fixed $0<\varepsilon<1$. More precisely, it is clear from (4) that

$$
D^{*}(n, \varepsilon)=\Phi^{-1}(1-\varepsilon)-\frac{\log n}{2 \sqrt{n V(X)}}+\mathrm{O}\left(\frac{1}{\sqrt{n}}\right)
$$

as $n \rightarrow \infty$ for fixed $0<\varepsilon<1$. By Lemma 3, Equation (47) can be extended to the case when $\varepsilon$ approaches to either zero or one polynomially in $n$ as follows: Given an arbitrary positive real number $r$, it follows from Lemmas 1 and 3 that

$$
\begin{aligned}
D^{*}\left(n, n^{-r}\right)= & \Phi^{-1}\left(1-n^{-r}\right)-\frac{(1+2 r) \log n-\log \log n}{2 \sqrt{n V(X)}} \\
& +\mathrm{O}\left(\frac{1}{\sqrt{n}}\right), \\
D^{*}\left(n, 1-n^{-r}\right)= & \Phi^{-1}\left(n^{-r}\right)-\frac{(1+2 r) \log n-\log \log n}{2 \sqrt{n V(X)}} \\
& +\mathrm{O}\left(\frac{1}{\sqrt{n}}\right)
\end{aligned}
$$

as $n \rightarrow \infty$. To asymptotically expand $\Phi^{-1}\left(1-n^{-r}\right)$ and $\Phi^{-1}\left(n^{-r}\right)$ in (48) and (49), respectively, we see from Lemma 1 that

$$
\begin{aligned}
D^{*}\left(n, n^{-r}\right)^{2}= & \Phi^{-1}\left(1-n^{-r}\right)^{2}+\mathrm{O}\left(\frac{\log ^{3 / 2} n}{\sqrt{n}}\right) \\
= & 2 r \ln n-\ln \frac{\pi}{2}-\ln (2 r \ln n-\ln \pi) \\
& +\mathrm{O}\left(\frac{\ln \ln n}{\ln n}\right)
\end{aligned}
$$

\footnotetext{
${ }^{7}$ The remainder term $+\mathrm{O}(1)$ in (45) depends only on $X$ and $r$, i.e., it is independent of the sequence $\left\{\varepsilon_{n}\right\}_{n=1}^{\infty}$.
}

as $n \rightarrow \infty$; and analogously, we get

$$
\begin{aligned}
D^{*}\left(n, 1-n^{-r}\right)^{2}= & \Phi^{-1}\left(n^{-r}\right)^{2}+\mathrm{O}\left(\frac{\log ^{3 / 2} n}{\sqrt{n}}\right) \\
= & 2 r \ln n-\ln \frac{\pi}{2}-\ln (2 r \ln n-\ln \pi) \\
& +\mathrm{O}\left(\frac{\ln \ln n}{\ln n}\right)
\end{aligned}
$$

as $n \rightarrow \infty$. Thus, we have obtained expressions for the higherorder optimal coding rates of the fixed-length compression problem when the error probabilities vanish polynomially in the blocklength $n$.

Our techniques to prove Lemma 3 involve using newlydeveloped moderate deviations results in Section V-A and strong large deviations results in Section V-B to analyze the fixed-length compression problem. These result in a new asymptotic expansion for fixed-length compression in Lemma 3 that is also amenable to integration over the error probability parameter (over a certain range) to obtain a thirdorder asymptotic expansion for the variable-length compression problem.

B. On the Cutoff Operation for Logarithm of Integer-Valued Random Variable

We now investigate a simplified version of the expectation in (28). The following two lemmas derive asymptotic expressions for $\mathbb{E}\left[\left\langle\log \gamma_{n}\left(X^{n}\right)\right\rangle_{\varepsilon}\right]$, i.e., the expectation of $\left\langle\log \gamma_{n}\left(X^{n}\right)\right\rangle_{\varepsilon}$ in the absence of the floor function noting that this operation does not affect the asymptotics.

Lemma 4. Given $0 \leq \varepsilon \leq 1$, it holds that

$$
\mathbb{E}\left[\left\langle\log \gamma_{n}\left(X^{n}\right)\right\rangle_{\varepsilon}\right]=\int_{\varepsilon}^{1} \log M^{*}(n, s) \mathrm{d} s+\mathrm{o}(1)
$$

as $n \rightarrow \infty$.

Proof of Lemma 4: See Appendix A.

Remark 3. The quantity $\mathbb{E}\left[\left\langle\log \gamma_{n}\left(X^{n}\right)\right\rangle_{\varepsilon}\right]$ that appears on the left-hand side of (52) is closely related to the fundamental limits of the guessing problem [37], [38] allowing errors [39] for a source X; see [34, Section IV].

Remark 4. In [7, Equations (44)-(48)], Szpankowski and Verdú showed that

$$
\mathbb{E}\left[\left\lfloor\log \gamma_{n}\left(X^{n}\right)\right\rfloor\right]=\int_{0}^{1}\left\lceil\log M^{*}(n, s)\right\rceil \mathrm{d} s-1,
$$

and the proof of Lemma 4 is similar to that of this identity. Note that $\left\lceil\log M^{*}(n, \varepsilon)\right\rceil$ denotes the infimum of integers $k$ such that an n-to-k binary block code for which the error probability is at most $\varepsilon$ exists. This quantity is slightly different from the fixed-length compression problem described in Section IV-A.

Lemma 5. Let $0<\varepsilon \leq 1$ be fixed. If the source $X$ satisfies Cramér's condition, then

$$
\begin{aligned}
& \mathbb{E}\left[\left\langle\log \gamma_{n}\left(X^{n}\right)\right\rangle_{\varepsilon}\right] \\
& =n(1-\varepsilon) H(X)-\sqrt{n V(X)} f_{\mathrm{G}}(\varepsilon)-\frac{1-\varepsilon}{2} \log n+\mathrm{O}(1)
\end{aligned}
$$


as $n \rightarrow \infty$. On the other hand, if $X$ is finitely supported, then

$$
\mathbb{E}\left[\log \gamma_{n}\left(X^{n}\right)\right]=n H(X)-\frac{1}{2} \log n+\mathrm{O}(1)
$$

as $n \rightarrow \infty$.

Proof of Lemma 5: The idea of the proof of Lemma 5 is to evaluate the integral in Lemma 4. For this purpose, we suppose that $X$ satisfies Cramér's condition. Since $\varepsilon \mapsto$ $\log M^{*}(n, \varepsilon)$ is nonnegative and nonincreasing on $(0,1)$, we readily see that

$$
\begin{gathered}
\int_{\varepsilon}^{1-n^{-1}} \log M^{*}(n, s) \mathrm{d} s \leq \int_{\varepsilon}^{1} \log M^{*}(n, s) \mathrm{d} s \\
\leq \int_{\varepsilon}^{1-n^{-1}} \log M^{*}(n, s) \mathrm{d} s+\frac{1}{n} \log M^{*}(n, \varepsilon)
\end{gathered}
$$

for $n \geq(1-\varepsilon)^{-1}$. Define

$$
\begin{aligned}
& K_{n}(X) \\
& :=\max _{n^{-1} \leq \varepsilon \leq 1-n^{-1}} \mid \log M^{*}(n, \varepsilon)-\left(n H(X)-\sqrt{n V(X)} \Phi^{-1}(\varepsilon)\right. \\
& \left.-\frac{1}{2} \log n+\left(S(X)-\frac{\log \mathrm{e}}{2}\right) \Phi^{-1}(\varepsilon)^{2}\right) \mid \text {. }
\end{aligned}
$$

Now, Lemma 3 implies that

$$
K_{n}(X)=\mathrm{O}(1)
$$

as $n \rightarrow \infty$. Then, it follows from Lemma 2 and (58) that

$$
\begin{aligned}
& \int_{\varepsilon}^{1-n^{-1}} \log M^{*}(n, s) \mathrm{d} s \\
& \leq n\left(1-\frac{1}{n}-\varepsilon\right) H(X)-\sqrt{n V(X)}\left(f_{\mathrm{G}}(\varepsilon)-f_{\mathrm{G}}\left(n^{-1}\right)\right) \\
& \quad-\frac{1-n^{-1}-\varepsilon}{2} \log n \\
& \quad-\frac{(\log \mathrm{e})\left(1-n^{-1}-\varepsilon-g_{\mathrm{G}}\left(1-n^{-1}\right)+g_{\mathrm{G}}(\varepsilon)\right)}{2}+K_{n}(X) \\
& =n(1-\varepsilon) H(X)-\sqrt{n V(X)} f_{\mathrm{G}}(\varepsilon)-\frac{1-\varepsilon}{2} \log n+\mathrm{O}(1) \quad(59)
\end{aligned}
$$

as $n \rightarrow \infty$. Analogously, we get

$$
\begin{aligned}
& \int_{\varepsilon}^{1-n^{-1}} \log M^{*}(n, s) \mathrm{d} s \\
& \geq n\left(1-\frac{1}{n}-\varepsilon\right) H(X)-\sqrt{n V(X)}\left(f_{\mathrm{G}}(\varepsilon)-f_{\mathrm{G}}\left(n^{-1}\right)\right) \\
& \quad-\frac{1-n^{-1}-\varepsilon}{2} \log n \\
& \quad-\frac{(\log \mathrm{e})\left(1-n^{-1}-\varepsilon-g_{\mathrm{G}}\left(1-n^{-1}\right)+g_{\mathrm{G}}(\varepsilon)\right)}{2}-K_{n}(X) \\
& =n(1-\varepsilon) H(X)-\sqrt{n V(X)} f_{\mathrm{G}}(\varepsilon)-\frac{1-\varepsilon}{2} \log n+\mathrm{O}(1)(60)
\end{aligned}
$$

as $n \rightarrow \infty$. Since $\log M^{*}(n, \varepsilon)=\mathrm{O}(n)$ as $n \rightarrow \infty$, combining (56), (59), and (60), we obtain (54) of Lemma 5.

Finally, suppose that $X$ is finitely supported. Similar to (56), we get

$$
\begin{aligned}
& \int_{n^{-1}}^{1-n^{-1}} \log M^{*}(n, s) \mathrm{d} s \leq \int_{0}^{1} \log M^{*}(n, s) \mathrm{d} s \\
& \leq \int_{n^{-1}}^{1-n^{-1}} \log M^{*}(n, s) \mathrm{d} s+2 \log |\operatorname{supp}(X)| .
\end{aligned}
$$

Since every finitely supported source $X$ satisfies Cramér's condition, it follows from Lemmas 2 and 3 that

$$
\begin{aligned}
& \int_{n^{-1}}^{1-n^{-1}} \log M^{*}(n, s) \mathrm{d} s=n\left(1-\frac{2}{n}\right) H(X)-\frac{1-2 n^{-1}}{2} \log n \\
& \quad-\frac{(\log \mathrm{e})\left(1-2 n^{-1}+2 g_{\mathrm{G}}\left(n^{-1}\right)\right)}{2}+\mathrm{O}(1) \\
& =n H(X)-\frac{1}{2} \log n+\mathrm{O}(1)
\end{aligned}
$$

as $n \rightarrow \infty$. Combining (61) and (62), we obtain (55) of Lemma 5. This completes the proof of Lemma 5.

We now use the above to complete the proof of Theorem 3. We see from (28) that (taking the floor operation into account)

$$
\left|\mathbb{E}\left[\left\langle\log \gamma_{n}\left(X^{n}\right)\right\rangle_{\varepsilon}\right]-L^{*}\left(\varepsilon \mid X^{n}\right)\right| \leq 1 .
$$

Therefore, Lemma 5 implies Theorem 3, completing the proof.

\section{Higher-ORdER Asymptotics OF FiXed-LENGTH COMPRESSION}

In this section, we prove Lemma 3 by employing certain variants of the moderate deviations and strong large deviations theorems. In the next two subsections, we introduce these fundamental results.

\section{A. Moderate Deviations}

Consider i.i.d. copies $\left\{Z_{i}\right\}_{i=1}^{\infty}$ of a real-valued r.v. $Z$ with zero mean. Suppose that the variance of $Z$,

$$
\sigma^{2}:=\mathbb{E}\left[Z^{2}\right],
$$

is positive and finite. Now, we want to characterize the distribution function defined by

$$
F_{n}(z):=\mathbb{P}\left\{\sum_{i=1}^{n} Z_{i} \leq z \sigma \sqrt{n}\right\}
$$

for each $z \in \mathbb{R}$. The central limit theorem states that

$$
F_{n}(z)=\Phi(z)+\mathrm{o}(1)
$$

uniformly on $\mathbb{R}$ as $n \rightarrow \infty$. In this study, to examine higher-order asymptotics of source coding problems either with vanishing error probabilities or with vanishing correct probabilities, we shall control the error term in (66) more precisely when $z$ diverges as $n \rightarrow \infty$. To this end, we shall use the following version of the moderate deviations theorem.

Lemma 6 ([12, Chapter VIII.2]). Suppose that the momentgenerating function $\mathbb{E}\left[\mathrm{e}^{t Z}\right]$ is finite for some neighborhood of $t=0$ (i.e., Cramér's condition on Z). Given a nonnegative real sequence $\left\{z_{n}\right\}_{n=1}^{\infty}$ satisfying $z_{n}=\mathrm{O}\left(n^{1 / 6}\right)$ as $n \rightarrow \infty$, it holds that

$$
\begin{aligned}
1-F_{n}\left(z_{n}\right) & =\left(1-\Phi\left(z_{n}\right)\right) \exp \left(\frac{S(Z) z_{n}^{3}}{\sqrt{n}}\right)+\mathrm{O}\left(\frac{\varphi\left(z_{n}\right)}{\sqrt{n}}\right), \\
F_{n}\left(-z_{n}\right) & =\Phi\left(-z_{n}\right) \exp \left(-\frac{S(Z) z_{n}^{3}}{\sqrt{n}}\right)+\mathrm{O}\left(\frac{\varphi\left(z_{n}\right)}{\sqrt{n}}\right)
\end{aligned}
$$

as $n \rightarrow \infty$, where $S(X)$ (one-sixth of the skewness) was defined in (10). 
Given a real number $0<\varepsilon<1$, choose $\zeta_{n}(\varepsilon) \in \mathbb{R}$ so that

$$
\zeta_{n}(\varepsilon):=\inf \left\{z \in \mathbb{R} \mid F_{n}(z) \geq 1-\varepsilon\right\}
$$

By (66), one readily sees

$$
\Phi\left(\zeta_{n}(\varepsilon)\right)=1-\varepsilon+\mathrm{o}(1)
$$

uniformly on $(0,1)$ as $n \rightarrow \infty$. We will, however, require a statement similar to (70) when $\varepsilon$ is a sequence $\left\{\varepsilon_{n}\right\}_{n=1}^{\infty}$ with limit infimum and limit supremum respectively equal to zero and one. The sequence $\left\{\varepsilon_{n}\right\}_{n=1}^{\infty}$ should also have the property that its subsequences approach zero or one polynomially fast. In fact, we will require a stronger statement that also quantifies the "rate of convergence".

Essentially, we are interested in "inverting" the moderate deviations result in Lemma 6. That is, suppose that $F_{n}\left(-z_{n}\right)=$ $\varepsilon_{n}$ where $F_{n}\left(-z_{n}\right)$ is the quantity having asymptotic expansion in (68), we would like to find how $z_{n}$ scales as $n \rightarrow \infty$. By the central limit theorem, we know that $z_{n}=-\Phi^{-1}\left(\varepsilon_{n}(1+\mathrm{o}(1))\right.$. We would like to carefully obtain a refinement of the o(1) term. In our application of these results, we will take $\varepsilon_{n}$ to be either $1 / n$ or $1-1 / n$ and thus, $z_{n} \approx \pm \sqrt{2 \ln n}$. Using Lemma 6 , we refine the $+o(1)$ term in (70) multiplicatively as follows:

Lemma 7. Let $\left\{\varepsilon_{n}\right\}_{n=1}^{\infty}$ be a real sequence satisfying ${ }^{8}$

$$
\frac{1}{n^{r}} \leq \varepsilon_{n} \leq \frac{1}{2}
$$

for some positive constant $r$. Suppose that the moment generating function $\mathbb{E}\left[\mathrm{e}^{t Z}\right]$ is finite for some neighborhood of $t=0$. Then, it holds that

$$
\begin{aligned}
& 1-\Phi\left(\zeta_{n}\left(\varepsilon_{n}\right)\right)=\varepsilon_{n}\left(1+\frac{S(Z) \Phi^{-1}\left(\varepsilon_{n}\right)^{3}}{\sqrt{n}}-\mathrm{O}\left(\frac{\Phi^{-1}\left(\varepsilon_{n}\right)}{\sqrt{n}}\right)\right) \\
& \Phi\left(\zeta_{n}\left(1-\varepsilon_{n}\right)\right)=\varepsilon_{n}\left(1-\frac{S(Z) \Phi^{-1}\left(\varepsilon_{n}\right)^{3}}{\sqrt{n}}+\mathrm{O}\left(\frac{\Phi^{-1}\left(\varepsilon_{n}\right)}{\sqrt{n}}\right)\right)
\end{aligned}
$$

as $n \rightarrow \infty$, where $S(Z)$ (one-sixth of the skewness) was defined in (10).

\section{Proof of Lemma 7: See Appendix B.}

Remark 5. Given a finitely supported nonlattice source $X$, let $Z=-\ln P_{X}(X)$. Then, for fixed $0<\varepsilon<1$, asymptotic expansions

$$
\Phi\left(\zeta_{n}(\varepsilon)\right)=1-\varepsilon+\mathrm{O}\left(n^{-1 / 2}\right)
$$

as $n \rightarrow \infty$ were investigated by Strassen [14, Equation (2.21)] based on the Edgeworth expansion (cf. [11, Chapter XVI.4] or [12, Chapter VI.3]). For a precise analysis of the BerryEsseen bound used to derive (74), we refer the reader to Kontoyiannis and Verdú's work [9, Section V]. On the other hand, Lemma 7 exhibits similar asymptotic expansions when either $\varepsilon_{n} \rightarrow 0^{+}$or $\varepsilon_{n} \rightarrow 1^{-}$along certain subsequences polynomially as $n \rightarrow \infty$.

\footnotetext{
${ }^{8}$ In our application of this result, we will take $\varepsilon_{n}=1 / n$ which satisfies (71) with $r=1$.
}

\section{B. Strong Large Deviations}

In this subsection, we introduce strong large deviations theorems for $\sigma$-finite measures that are not necessarily probability measures. ${ }^{9}$ Let $(\Omega, \mathcal{F}, \mu)$ be a $\sigma$-finite measure space, and $f: \Omega \rightarrow \mathbb{R}$ a Borel-measurable function. Denote by $\mu_{f}:=\mu \circ f^{-1}$ the measure on $\mathbb{R}$ induced by $f$. Define the cumulant generating function as

$$
\Lambda_{\mu_{f}}(s):=\ln \left(\int_{\mathbb{R}} \mathrm{e}^{s t} \mu_{f}(\mathrm{~d} t)\right),
$$

and the Fenchel-Legendre transform of $\Lambda_{\mu_{f}}(s)$ by

$$
\Lambda_{\mu_{f}}^{*}(a):=\sup _{s \in \mathbb{R}}\left(a s-\Lambda_{\mu_{f}}(s)\right) .
$$

Let $\mathcal{D}_{\mu_{f}}:=\left\{s \mid \Lambda_{\mu_{f}}(s)<\infty\right\}$ and $\operatorname{int}\left(\mathcal{D}_{\mu_{f}}\right)$ its interior. Similar to [13, Lemma 2.2.5 and Exercise 2.2.24], it can be verified by Hölder's inequality and the dominated convergence theorem for the Lebesgue integrals with respect to a $\sigma$ finite measure that $\Lambda_{\mu_{f}}(s)$ is of class $C^{\infty}$ in $s \in \operatorname{int}\left(\mathcal{D}_{\mu_{f}}\right)$. Especially, it holds that for each $s \in \operatorname{int}\left(\mathcal{D}_{\mu_{f}}\right)$,

$$
\Lambda_{\mu_{f}}^{\prime}(s)=a \quad \Longrightarrow \quad \Lambda_{\mu_{f}}^{*}(a)=a s-\Lambda_{\mu_{f}}(s) .
$$

Similar to Section II-A, one can consider the notion of $\mu_{f}$ being a lattice measure. We say that $\mu_{f}$ is a lattice measure if $\mu_{f}$ is discrete ${ }^{10}$ and there exists a positive constant $d$ such that $f\left(t_{1}\right)-f\left(t_{2}\right)$ is a multiple of $d$ whenever $\mu_{f}\left(t_{1}\right) \mu_{f}\left(t_{2}\right)>0$. Otherwise, we say that $\mu_{f}$ is a nonlattice measure. For a lattice measure $\mu_{f}$, its maximal span $d_{f}$ is defined by the maximum of those $d$. For convenience, we set $d_{f}=0$ if $\mu_{f}$ is nonlattice. Then, given a positive parameter $s$, define

$$
v_{s}(f):= \begin{cases}\frac{d_{f}}{\mathrm{e}^{d_{f} s}-1} & \text { if } \mu_{f} \text { is lattice, } \\ s^{-1} & \text { if } \mu_{f} \text { is nonlattice. }\end{cases}
$$

Now, consider $n$ Borel-measurable functions $f_{1}, \ldots, f_{n}$ in which $\mu \circ\left(f_{1}, \ldots, f_{n}\right)^{-1}=\mu_{f_{1}} \times \cdots \times \mu_{f_{n}}$ and $\mu_{f_{i}}=\mu_{f}$ for each $1 \leq i \leq n .{ }^{11}$ The following lemma states a strong large deviations result known as the Bahadur-Rao theorem.

Lemma 8 ([13, Theorem 3.7.4] and [12, Chapter VIII.4] for probability measures $\mu$ ). Let $a=\Lambda_{\mu_{f}}^{\prime}(s)$ for some positive $s \in \operatorname{int}\left(\mathcal{D}_{\mu_{f}}\right)$. Then, it holds that ${ }^{12}$

$$
\begin{aligned}
& \mu\left\{\sum_{i=1}^{n} f_{i}>a n\right\}=\frac{\mathrm{e}^{-n \Lambda_{\mu_{f}}^{*}(a)}}{\sqrt{2 \pi n \Lambda_{\mu_{f}}^{\prime \prime}(s)}}\left(v_{s}(f)+\mathrm{o}(1)\right), \\
& \mu\left\{\sum_{i=1}^{n} f_{i}=a n\right\}=\frac{\mathrm{e}^{-n \Lambda_{\mu_{f}}^{*}(a)}}{\sqrt{2 \pi n \Lambda_{\mu_{f}}^{\prime \prime}(s)}}\left(d_{f}+\mathrm{o}(1)\right) .
\end{aligned}
$$

${ }^{9}$ For strong large deviations for finite measures, refer to [20, Section VIII] or [40, Footnote 8]. In this study, we consider $\sigma$-finite measures to deal with a countably infinite source alphabet $\mathcal{X}$, because the results on finite measures are applicable only for finite source alphabets.

${ }^{10} \mathrm{~A}$ measure $v$ is said to be discrete if there exists a measurable set $\mathcal{E}$ such that it is countable and $v\left(\mathcal{E}^{\complement}\right)=0$, where $\mathcal{E}^{\complement}$ denotes the complement of $\mathcal{E}$.

${ }^{11}$ When $\mu$ is a probability measure, this implies that $f_{1}, \ldots, f_{n}$ are i.i.d. copies of a real-valued r.v. $f$.

${ }^{12}$ When $\mu_{f}$ is lattice, then the remainder terms +o(1) can be refined as $+\mathrm{O}\left(n^{-1}\right)$; see [12, Chapter VIII.4]. 
In [13, Theorem 3.7.4] and [12, Chapter VIII.4], Lemma 8 is stated in the case when $\mu$ is a probability measure, and its proof can be readily extended to $\sigma$-finite measures $\mu$. We give a proof sketch of Lemma 8 in Appendix C.

The following lemma is a variant of Lemma 8.

Lemma 9. Let $\left\{\mathcal{I}_{n}\right\}_{n=1}^{\infty}$ be a sequence of real intervals, and $\mathcal{I}=\bigcup_{n} \mathcal{I}_{n}$. Consider a real function $a_{n}(\cdot)$ on $\mathcal{I}$ for each $n \in \mathbb{N}$. Suppose that $a=\Lambda_{\mu_{f}}^{\prime}(s)$ for some positive $s \in \operatorname{int}\left(\mathcal{D}_{\mu_{f}}\right)$. If

$$
a_{n}(t)=\mathrm{o}(n)
$$

uniformly on $\mathcal{I}_{n}$ as $n \rightarrow \infty$, then there exist $r_{f}^{(1)}(n, t)=\mathrm{o}(1)$ and $r_{f}^{(2)}(n, t)=\mathrm{O}(1)$ uniformly on $\mathcal{I}_{n}$ as $n \rightarrow \infty$ such that

$$
\begin{aligned}
& \mu\left\{\sum_{i=1}^{n} f_{i}>a n+a_{n}(t)\right\} \\
& =\frac{\mathrm{e}^{-K_{f}(n, s, t)}}{\sqrt{2 \pi n \Lambda_{\mu_{f}}^{\prime \prime}(s)(1+\mathrm{o}(1))}}\left(v_{s}(f)+\mathrm{o}(1)\right), \\
& \mu\left\{\sum_{i=1}^{n} f_{i}=a n+a_{n}(t)\right\} \\
& =\frac{\mathrm{e}^{-K_{f}(n, s, t)}}{\sqrt{2 \pi n \Lambda_{\mu_{f}}^{\prime \prime}(s)(1+\mathrm{o}(1))}}\left(d_{f}+\mathrm{o}(1)\right)
\end{aligned}
$$

uniformly on $\mathcal{I}_{n}$ as $n \rightarrow \infty$, where the exponent part $K_{f}(n, s, t)$ is given as

$$
K_{f}(n, s, t)=n \Lambda_{\mu_{f}}^{*}(a)+s a_{n}(t)+\frac{a_{n}(t)^{2}}{2 n \Lambda_{\mu_{f}}^{\prime \prime}(s)}(1+o(1))
$$

uniformly on $\mathcal{I}_{n}$ as $n \rightarrow \infty$.

$$
\text { Proof of Lemma 9: See Appendix D. }
$$

Remark 6. Lemma 9 is a minor extension of Hayashi's technical result [20, Lemma 3]. The main differences vis-àvis [20, Lemma 3] is that the asymptotic expansion in (84) is refined.

\section{Proof of Lemma 3}

Denote by $\iota_{n}(X):=-\ln P_{X^{n}}\left(X^{n}\right)$ the information density of $X^{n}$, where $\iota(X):=\iota_{1}(X)$. Consider a $\sigma$-finite measure $\mu$ in which $\mu_{X}$ is the counting measure on $\operatorname{supp}(X)$ and $\mu_{X}(X \backslash \operatorname{supp}(X))=0$. Now, define $v_{X}:=\mu \circ(-\iota(X))^{-1}$. Since $H_{\alpha}(X)<\infty$ for some $0<\alpha<1$, we observe that $s \mapsto \Lambda_{v_{X}}(s)$ is infinitely differentiable at $s=1$. Then, a direct calculation shows

$$
\begin{aligned}
& \Lambda_{v_{X}}^{\prime}(1)=-(\ln 2) H(X), \\
& \Lambda_{v_{X}}^{\prime \prime}(1)=(\ln 2)^{2} V(X) .
\end{aligned}
$$

Choose the nonnegative number $\eta_{n}\left(\varepsilon_{n}, X\right)$ so that

$$
\begin{aligned}
& F_{n}^{+}\left(\varepsilon_{n}, X\right):=\mathbb{P}\left\{\iota_{n}(X) \leq \eta_{n}\left(\varepsilon_{n}, X\right)\right\} \geq 1-\varepsilon_{n}, \\
& F_{n}^{-}\left(\varepsilon_{n}, X\right):=\mathbb{P}\left\{\iota_{n}(X)<\eta_{n}\left(\varepsilon_{n}, X\right)\right\}<1-\varepsilon_{n},
\end{aligned}
$$

respectively. It follows from (43) that $^{13}$

$$
\begin{aligned}
& M^{*}\left(n, \varepsilon_{n}\right) \\
& =\mu\left\{\iota_{n}(X)<\eta_{n}\left(\varepsilon_{n}, X\right)\right\} \\
& \quad+\left\lceil\left(\frac{\left(1-\varepsilon_{n}\right)-F_{n}^{-}\left(\varepsilon_{n}, X\right)}{F_{n}^{+}\left(\varepsilon_{n}, X\right)-F_{n}^{-}\left(\varepsilon_{n}, X\right)}\right) \mu\left\{\iota_{n}(X)=\eta_{n}\left(\varepsilon_{n}, X\right)\right\}\right],
\end{aligned}
$$

yielding that

$\mu\left\{\iota_{n}(X)<\eta_{n}\left(\varepsilon_{n}, X\right)\right\}<M^{*}\left(n, \varepsilon_{n}\right) \leq \mu\left\{\iota_{n}(X) \leq \eta_{n}\left(\varepsilon_{n}, X\right)\right\}$.

Fix a positive number $r$ arbitrarily. Define

$$
\lambda_{n}\left(\varepsilon_{n}, X\right):=\frac{\eta_{n}\left(\varepsilon_{n}, X\right)-n(\ln 2) H(X)}{(\ln 2) \sqrt{n V(X)}} .
$$

By Taylor's theorem for $s \mapsto \Phi^{-1}(s)$ around $s=1-\varepsilon_{n}$, we observe that

$$
\lambda_{n}\left(\varepsilon_{n}, X\right)=\Phi^{-1}\left(1-\varepsilon_{n}\right)-\frac{\left(1-\varepsilon_{n}\right)-\Phi\left(\lambda_{n}\left(\varepsilon_{n}, X\right)\right)}{f_{\mathrm{G}}\left(s_{n}\left(\varepsilon_{n}, X\right)\right)},
$$

where $0<s_{n}\left(\varepsilon_{n}, X\right)<1$ is given by

$s_{n}\left(\varepsilon_{n}, X\right):=\theta_{n}\left(\varepsilon_{n}, X\right)\left(1-\varepsilon_{n}\right)+\left(1-\theta_{n}\left(\varepsilon_{n}, X\right)\right) \Phi\left(\lambda_{n}\left(\varepsilon_{n}, X\right)\right)$

for some $0 \leq \theta_{n}\left(\varepsilon_{n}, X\right) \leq 1$. Substituting (92) into (91), we see that

$$
\begin{aligned}
& \frac{\eta_{n}\left(\varepsilon_{n}, X\right)}{\ln 2} \\
& =n H(X)-\sqrt{n V(X)}\left(\Phi^{-1}\left(\varepsilon_{n}\right)+\frac{\left(1-\varepsilon_{n}\right)-\Phi\left(\lambda_{n}\left(\varepsilon_{n}, X\right)\right)}{f_{\mathrm{G}}\left(s_{n}\left(\varepsilon_{n}, X\right)\right)}\right) .
\end{aligned}
$$

Now, suppose that (71) holds. Then, we see from (72) of Lemma 7 that

$$
s_{n}\left(\varepsilon_{n}, X\right) \leq 1-\varepsilon_{n}\left(1+\frac{S(X) \Phi^{-1}\left(\varepsilon_{n}\right)^{3}}{\sqrt{n}}-\mathrm{O}\left(\frac{\Phi^{-1}\left(\varepsilon_{n}\right)}{\sqrt{n}}\right)\right) \text {. }
$$

for sufficiently large $n$. Similarly, we get that

$$
s_{n}\left(\varepsilon_{n}, X\right) \geq 1-\varepsilon_{n}\left(1-\frac{S(X) \Phi^{-1}\left(\varepsilon_{n}\right)^{3}}{\sqrt{n}}+\mathrm{O}\left(\frac{\Phi^{-1}\left(\varepsilon_{n}\right)}{\sqrt{n}}\right)\right) .
$$

for sufficiently large $n$. It follows from (15), (16), and (71) that

$$
\left|\frac{S(X) \Phi^{-1}\left(\varepsilon_{n}\right)^{3}}{\sqrt{n}}-\mathrm{O}\left(\frac{\Phi^{-1}\left(\varepsilon_{n}\right)}{\sqrt{n}}\right)\right| \leq \mathrm{O}\left(\sqrt{\frac{\ln ^{3} n}{n}}\right) .
$$

as $n \rightarrow \infty$. Inserting this estimate into (95) and (96), we obtain

$$
s_{n}\left(\varepsilon_{n}, X\right)=1-\varepsilon_{n}\left(1+\mathrm{O}\left(\sqrt{\frac{\ln ^{3} n}{n}}\right)\right)
$$

as $n \rightarrow \infty$. Therefore, it follows from (16) and Taylor's theorem for $s \mapsto f_{\mathrm{G}}(s)$ around $s=1-\varepsilon_{n}$ that there exists

\footnotetext{
${ }^{13}$ This identity is a consequence of the Neyman-Pearson lemma.
} 
some $a_{n}$ between $1-\varepsilon_{n}$ and $1-\varepsilon_{n}+\mathrm{O}\left(\left(n^{-1} \ln n\right)^{1 / 2}\right)$ such that

$$
\begin{aligned}
f_{\mathrm{G}}\left(s_{n}\left(\varepsilon_{n}, X\right)\right) & =f_{\mathrm{G}}\left(1-\varepsilon_{n}\right)+\mathrm{O}\left(f_{\mathrm{G}}^{\prime}\left(a_{n}\right) \varepsilon_{n} \sqrt{\frac{\ln ^{3} n}{n}}\right) \\
& =f_{\mathrm{G}}\left(\varepsilon_{n}\right)+\mathrm{O}\left(\Phi^{-1}\left(a_{n}\right) \varepsilon_{n} \sqrt{\frac{\ln ^{3} n}{n}}\right) \\
& =f_{\mathrm{G}}\left(\varepsilon_{n}\right)+\mathrm{O}\left(\frac{\varepsilon_{n} \ln ^{2} n}{\sqrt{n}}\right) \\
& =f_{\mathrm{G}}\left(\varepsilon_{n}\right)(1+\mathrm{o}(1))
\end{aligned}
$$

as $n \rightarrow \infty$. On the other hand, from (72) of Lemma 7,

$$
\left|\left(1-\varepsilon_{n}\right)-\Phi\left(\lambda_{n}\left(\varepsilon_{n}, X\right)\right)\right| \leq \varepsilon_{n}\left|\frac{S(X) \Phi^{-1}\left(\varepsilon_{n}\right)^{3}}{\sqrt{n}}-\mathrm{O}\left(\frac{\Phi^{-1}\left(\varepsilon_{n}\right)}{\sqrt{n}}\right)\right| .
$$

for sufficiently large $n$. Combining (99) and (100), we have from (15) and (16) that

$$
\begin{aligned}
\left|\frac{\left(1-\varepsilon_{n}\right)-\Phi^{-1}\left(\lambda_{n}\left(\varepsilon_{n}, X\right)\right)}{f_{\mathrm{G}}\left(s_{n}\left(\varepsilon_{n}, X\right)\right)}\right| & \leq \varepsilon_{n}\left|\frac{\frac{S(X) \Phi^{-1}\left(\varepsilon_{n}\right)^{3}}{\sqrt{n}}-\mathrm{O}\left(\frac{\Phi^{-1}\left(\varepsilon_{n}\right)}{\sqrt{n}}\right)}{f_{\mathrm{G}}\left(\varepsilon_{n}\right)(1+\mathrm{o}(1))}\right| \\
& =\frac{1}{\sqrt{n}}\left(S(X) \Phi^{-1}\left(\varepsilon_{n}\right)^{2}+\mathrm{O}(1)\right),
\end{aligned}
$$

as $n \rightarrow \infty$. Hence, it follows from (94) and (101) that

$$
\begin{array}{rl}
\frac{\eta_{n}\left(\varepsilon_{n}, X\right)}{\ln 2}=n & H(X)-\sqrt{n V(X)} \Phi^{-1}\left(\varepsilon_{n}\right) \\
& +\left(S(X) \Phi^{-1}\left(\varepsilon_{n}\right)^{2}+\mathrm{O}(1)\right)
\end{array}
$$

as $n \rightarrow \infty$, provided that (71) holds. By using (73) of Lemma 7 rather than (72), we can prove (102) in an analogous manner even if the sequence $\left\{\varepsilon_{n}\right\}_{n=1}^{\infty}$ satisfies that

$$
\frac{1}{2} \leq \varepsilon_{n} \leq 1-\frac{1}{n^{r}} \text {. }
$$

Therefore, we conclude that (102) holds for every sequence $\left\{\varepsilon_{n}\right\}_{n=1}^{\infty}$ satisfying (44). Now, note from (85) and (86) that (102) can be rewritten as

$$
\begin{aligned}
\eta_{n}\left(\varepsilon_{n}, X\right)=- & n \Lambda_{v_{X}}^{\prime}(1)-\sqrt{n \Lambda_{v_{X}}^{\prime \prime}(1)} \Phi^{-1}\left(\varepsilon_{n}\right) \\
+ & \left(\Phi^{-1}\left(\varepsilon_{n}\right)^{2} S(X)+\mathrm{O}(1)\right)
\end{aligned}
$$

as $n \rightarrow \infty$. Therefore, applying (82) of Lemma 9 with

$$
\begin{aligned}
s & =1, \\
f_{i} & =\ln P_{X_{i}}\left(X_{i}\right) \quad(\text { for } i=1, \ldots, n), \\
a_{n}\left(\varepsilon_{n}\right) & =\sqrt{n \Lambda_{v_{X}}^{\prime \prime}(1)} \Phi^{-1}\left(\varepsilon_{n}\right)+\mathrm{O}(1)
\end{aligned}
$$

we obtain (108) (at the top of the next page) as $n \rightarrow \infty$, where $v(X)$ is given as

$$
v(X):= \begin{cases}\frac{(\ln 2) d_{X}}{2^{d_{X}}-1} & \text { if } X \text { is a lattice source } \\ 1 & \text { if } X \text { is a nonlattice source }\end{cases}
$$

and $d_{X}$ is defined in Section II-A. Analogously, we get from (83) of Lemma 9 that

$$
\begin{aligned}
& \mu\left\{\iota_{n}(X)=\eta_{n}\left(\varepsilon_{n}, X\right)\right\} \\
& =\frac{(\ln 2) d_{X}+\mathrm{o}(1)}{\sqrt{2 \pi n(\ln 2)^{2} V(X)(1+\mathrm{o}(1))}} \\
& \quad \times \exp \left(\eta_{n}\left(\varepsilon_{n}, X\right)+\left(S(X)-\frac{1}{2}\right) \Phi^{-1}\left(\varepsilon_{n}\right)^{2}+\mathrm{O}(1)\right)
\end{aligned}
$$

as $n \rightarrow \infty$. Combining (90), (108), and (110), we obtain

$$
\begin{aligned}
& \ln M^{*}\left(n, \varepsilon_{n}\right) \\
& =\eta_{n}\left(\varepsilon_{n}, X\right)+\left(S(X)-\frac{1}{2}\right) \Phi^{-1}\left(\varepsilon_{n}\right)^{2} \\
& \quad \quad-\frac{1}{2} \ln \left(2 \pi n(\ln 2)^{2} V(X)(1+o(1))\right)+\mathrm{O}(1) \\
& =\eta_{n}\left(\varepsilon_{n}, X\right)-\frac{1}{2} \ln n+\left(S(X)-\frac{1}{2}\right) \Phi^{-1}\left(\varepsilon_{n}\right)^{2}+\mathrm{O}(1)
\end{aligned}
$$

as $n \rightarrow \infty$. This completes the proof of Lemma 3 .

\section{CONCLuding Remarks AND Future Works}

In this study, we investigated the third-order asymptotics of the problem of variable-length compression allowing errors. Our main contribution is in refining the second-order asymptotic expansion of Kostina, Polyanskiy, and Verdú [8] to obtain the third-order term which is $-(1-\varepsilon)(\log n) / 2$ where $\varepsilon$ is the permissible error probability in reconstructing the source. Our proof strategy demonstrates a novel utility of a combination of moderate deviations (or Cramér-type large deviations) and strong large deviations analyses in information theory.

One extension of the work herein is to consider the thirdorder term in the classical channel coding problem [17], [41], [42] in the moderate deviations regime, i.e., the analogue of Lemma 3 for the channel coding setting. By evaluating Polyanskiy, Poor, Verdú's random coding union (RCU) bound [17, Theorem 16] and the meta-converse [17, Theorem 27] with carefully chosen output distributions (e.g., in [42]), and replacing the use of the Berry-Esseen theorem with Lemma 6 and 7 of the present paper, this should yield the third-order term in the moderate deviations regime. Such a strategy may also be amenable to additive white Gaussian noise (AWGN) channels [41].

\section{APPENDIX A \\ PROOF OF LEMMA 4}

To prove Lemma 4, we first choose a positive integer $\xi_{n}=$ $\xi_{n}(n, X)$ so that ${ }^{14}$

$$
\begin{aligned}
& \mathbb{P}\left\{\gamma_{n}\left(X^{n}\right) \geq \xi_{n}\right\} \geq \varepsilon, \\
& \mathbb{P}\left\{\gamma_{n}\left(X^{n}\right)>\xi_{n}\right\}<\varepsilon
\end{aligned}
$$

for each $0<\varepsilon \leq 1$, and $\xi_{n}=2^{n H_{0}(X)}$ if $\varepsilon=0$, where

$$
H_{0}(X):= \begin{cases}\log |\operatorname{supp}(X)| & \text { if } \operatorname{supp}(X) \text { is finite, } \\ \infty & \text { if } \operatorname{supp}(X) \text { is infinite. }\end{cases}
$$

${ }^{14}$ Note that $\xi_{n}$ plays the role of $\tilde{\xi}_{n}$ defined in (38)-(39). In fact, it is clear that $\xi_{n}=\log \tilde{\xi}_{n}$. 


$$
\begin{aligned}
& \mu\left\{\iota_{n}(X)<\eta_{n}\left(\varepsilon_{n}, X\right)\right\} \\
& =\mu\left\{\sum_{i=1}^{n} \ln P_{X_{i}}\left(X_{i}\right)>n \Lambda_{v_{X}}^{\prime}(1)+\sqrt{n \Lambda_{v_{X}}^{\prime \prime}(1)} \Phi^{-1}\left(\varepsilon_{n}\right)+\mathrm{O}(1)\right\} \\
& =\frac{v(X)+\mathrm{o}(1)}{\sqrt{2 \pi n \Lambda_{v_{X}}^{\prime \prime}(1)(1+\mathrm{o}(1))}} \exp \left(-n \Lambda_{v_{X}}^{*}\left(\Lambda_{v_{X}}^{\prime}(1)\right)-\left(\sqrt{n \Lambda_{v_{X}}^{\prime \prime}(1)} \Phi^{-1}\left(\varepsilon_{n}\right)+\mathrm{O}(1)\right)\right. \\
& \left.-\frac{1}{2 n \Lambda_{\nu_{X}}^{\prime \prime}(1)}\left(\sqrt{n \Lambda_{\nu_{X}}^{\prime \prime}(1)} \Phi^{-1}\left(\varepsilon_{n}\right)+\mathrm{O}(1)\right)^{2}\right) \\
& =\frac{v(X)+\mathrm{o}(1)}{\sqrt{2 \pi n(\ln 2)^{2} V(X)(1+\mathrm{o}(1))}} \exp \left(n(\ln 2) H(X)-(\ln 2) \sqrt{n V(X)} \Phi^{-1}\left(\varepsilon_{n}\right)+\left(S(X)-\frac{1}{2}\right) \Phi^{-1}\left(\varepsilon_{n}\right)^{2}+\mathrm{O}(1)\right) \\
& =\frac{v(X)+\mathrm{o}(1)}{\sqrt{2 \pi n(\ln 2)^{2} V(X)(1+\mathrm{o}(1))}} \exp \left(\eta_{n}\left(\varepsilon_{n}, X\right)+\left(S(X)-\frac{1}{2}\right) \Phi^{-1}\left(\varepsilon_{n}\right)^{2}+\mathrm{O}(1)\right)
\end{aligned}
$$

It is clear that $\xi_{n}=2^{n H_{\infty}(X)}$ if $\varepsilon=1$, where $H_{\infty}(X):=-\log \max _{x \in \mathcal{X}} P_{X}(x)$ denotes the min-entropy. Since $\mathbb{E}\left[\left\langle\log \gamma_{n}\left(X^{n}\right)\right\rangle_{\varepsilon}\right]=0$ if $\varepsilon \geq 1-2^{-n H_{\infty}(X)}$, it suffices to consider the case when $0 \leq \varepsilon<1-2^{-n H_{\infty}(X)}$. Then, a direct calculation shows that

$$
\begin{aligned}
& \mathbb{E}\left[\left\langle\log \gamma_{n}\left(X^{n}\right)\right\rangle_{\varepsilon}\right] \\
& \stackrel{(a)}{=} \int_{0}^{\infty} \mathbb{P}\left\{\left\langle\log \gamma_{n}\left(X^{n}\right)\right\rangle_{\varepsilon}>s\right\} \mathrm{d} s \\
& \stackrel{\text { (b) }}{\leq} \int_{0}^{\log \xi_{n}} \mathbb{P}\left\{s<\log \gamma_{n}\left(X^{n}\right) \leq \log \xi_{n}\right\} \mathrm{d} s \\
& \stackrel{\text { (c) }}{=} \int_{0}^{\infty} \mathbb{P}\left\{\log \gamma_{n}\left(X^{n}\right)>s\right\} \mathrm{d} s-\int_{\log \xi_{n}}^{\infty} \mathbb{P}\left\{\log \gamma_{n}\left(X^{n}\right)>t\right\} \mathrm{d} t \\
& -\left(\log \xi_{n}\right) \mathbb{P}\left\{\gamma_{n}\left(X^{n}\right)>\xi_{n}\right\} \\
& =\int_{0}^{\infty} \mathbb{P}\left\{\log \gamma_{n}\left(X^{n}\right)>s\right\} \mathrm{d} s-\int_{\log \xi_{n}}^{\log \left(\xi_{n}+1\right)} \mathbb{P}\left\{\log \gamma_{n}\left(X^{n}\right)>t\right\} \mathrm{d} t \\
& -\sum_{k=\xi_{n}+1}^{\infty} \int_{\log k}^{\log (k+1)} \mathbb{P}\left\{\log \gamma_{n}\left(X^{n}\right)>u\right\} \mathrm{d} u \\
& -\left(\log \xi_{n}\right) \mathbb{P}\left\{\gamma_{n}\left(X^{n}\right)>\xi_{n}\right\} \\
& =\int_{0}^{\infty} \mathbb{P}\left\{\log \gamma_{n}\left(X^{n}\right)>s\right\} \mathrm{d} s \\
& -\left(\log \left(\xi_{n}+1\right)-\log \xi_{n}\right) \mathbb{P}\left\{\log \gamma_{n}\left(X^{n}\right)>\log \xi_{n}\right\} \\
& \text { - } \sum_{k=\xi_{n}+1}^{\infty}(\log (k+1)-\log k) \mathbb{P}\left\{\log \gamma_{n}\left(X^{n}\right)>\log k\right\} \\
& -\left(\log \xi_{n}\right) \mathbb{P}\left\{\gamma_{n}\left(X^{n}\right)>\xi_{n}\right\} \\
& =\int_{0}^{\infty} \mathbb{P}\left\{\log \gamma_{n}\left(X^{n}\right)>s\right\} \mathrm{d} s-\sum_{k=1+\xi_{n}}^{\infty}(\log k) \mathbb{P}\left\{\gamma_{n}\left(X^{n}\right)=k\right\}
\end{aligned}
$$$$
=\sum_{k=2}^{\xi_{n}}(\log k) \mathbb{P}\left\{\gamma_{n}\left(X^{n}\right)=k\right\}
$$$$
=\sum_{k=2}^{\xi_{n}}(\log k) \int_{\mathbb{P}\left\{\gamma_{n}\left(X^{n}\right)>k\right\}}^{\mathbb{P}\left\{\gamma_{n}\left(X^{n}\right) \geq k\right\}} \mathrm{d} s
$$$$
\stackrel{(\mathrm{d})}{=} \int_{\mathbb{P}\left\{\gamma_{n}\left(X^{n}\right)>\xi_{n}\right\}}^{1-2^{-n H_{\infty}(X)}} \log \left(M^{*}(n, s)+1\right) \mathrm{d} s
$$

$$
\begin{aligned}
& =\int_{\varepsilon}^{1-2^{-n H_{\infty}(X)}} \log \left(M^{*}(n, s)+1\right) \mathrm{d} s \\
& +\left(\varepsilon-\mathbb{P}\left\{\gamma_{n}\left(X^{n}\right)>\xi_{n}\right\}\right) \log \left(M^{*}(n, \varepsilon)+1\right) \\
& \stackrel{\text { (e) }}{\leq} \int_{\varepsilon}^{1-2^{-n H_{\infty}(X)}} \log \left(M^{*}(n, s)+1\right) \mathrm{d} s \\
& \mathbb{P}\left\{\gamma_{n}\left(X^{n}\right)=\xi_{n}\right\} \log \left(M^{*}(n, \varepsilon)+1\right) \\
& \stackrel{\text { (f) }}{\leq} \int_{\varepsilon}^{1-2^{-n H_{\infty}(X)}} \log \left(M^{*}(n, s)+1\right) \mathrm{d} s \\
& +2^{-n H_{\infty}(X)} \log \left(M^{*}(n, \varepsilon)+1\right),
\end{aligned}
$$

where

- (a) follows from the fact that $\mathbb{E}[Z]=\int_{0}^{\infty} \mathbb{P}\{Z>z\} \mathrm{d} z$ for every nonnegative-real-valued r.v. $Z$,

- (b) follows from (113),

- (c) follows by the following elementary calculation

$$
\begin{aligned}
& \int_{0}^{a} \mathbb{P}\{s<Y \leq a\} \mathrm{d} s+a \mathbb{P}\{Y>a\}+\int_{a}^{\infty} \mathbb{P}\{Y>s\} \mathrm{d} s \\
& =\int_{0}^{a} \mathbb{P}\{s<Y \leq a\} \mathrm{d} s+\int_{0}^{a} \mathbb{P}\{Y>a\} \mathrm{d} s+\int_{a}^{\infty} \mathbb{P}\{Y>s\} \mathrm{d} s \\
& =\int_{0}^{a} \mathbb{P}\{Y>s\} \mathrm{d} s+\int_{a}^{\infty} \mathbb{P}\{Y>s\} \mathrm{d} s=\int_{0}^{\infty} \mathbb{P}\{Y>s\} \mathrm{d} s
\end{aligned}
$$

and taking $Y=\log \gamma_{n}\left(X^{n}\right)$ and $a=\log \xi_{n}$,

- (d) follows from the fact that $M^{*}(n, s)=k-1$ if $\mathbb{P}\left\{\gamma_{n}\left(X^{n}\right)>k\right\}<s<\mathbb{P}\left\{\gamma_{n}\left(X^{n}\right) \geq k\right\}$,

- (e) follows from (112), and

- (f) follows from the fact that

$$
2^{-n H_{\infty}(X)}=\mathbb{P}\left\{\gamma_{n}\left(X^{n}\right)=1\right\} \geq \mathbb{P}\left\{\gamma_{n}\left(X^{n}\right)=k\right\}
$$

for every integer $k \geq 1$.

Analogously, we see that

$$
\begin{aligned}
& \mathbb{E}\left[\left\langle\log \gamma_{n}\left(X^{n}\right)\right\rangle_{\varepsilon}\right] \\
& \quad \geq \int_{\varepsilon}^{1} \log \left(M^{*}(n, s)-1\right) \mathrm{d} s-2^{-n H_{\infty}(X)} \log \left(M^{*}(n, \varepsilon)-1\right)
\end{aligned}
$$

Combining (126) and (129) and using the fact that $\log M^{*}(n, s)$ is $\Theta(n)$, we obtain Lemma 4. 


\section{APPENDIX B}

\section{PROOF OF LEMMA 7}

For the sake of brevity, we use $\mathrm{Q}(z):=1-\Phi(z)=\Phi(-z)$, the complementary Gaussian cumulative distribution function, in this proof. In the following, we only prove (73); the proof for (72) follows analogously. Starting from (68) in Lemma 6, we have

$$
F_{n}\left(-z_{n}\right)=\mathrm{Q}\left(z_{n}\right) \exp \left(-\frac{z_{n}^{3} S(Z)}{\sqrt{n}}\right)+\mathrm{O}\left(\frac{1}{\sqrt{n}} \exp \left(-\frac{z_{n}^{2}}{2}\right)\right),
$$

where the final term (involving $\mathrm{O}(\cdot)$ ) results from the definition of the Gaussian probability density function $\varphi(u)=\frac{1}{\sqrt{2 \pi}} \mathrm{e}^{-u^{2} / 2}$. We set this to be equal to $\varepsilon_{n}$ to solve for $z_{n}$, i.e.,

$$
F_{n}\left(-z_{n}\right)=\varepsilon_{n}
$$

From the usual central limit theorem (70), we deduce that $z_{n}=\mathrm{Q}^{-1}\left(\varepsilon_{n}(1+\mathrm{o}(1))\right)$. Thus, we can parametrize it as

$$
z_{n}=\mathrm{Q}^{-1}\left(\varepsilon_{n} \exp \left(g\left(\varepsilon_{n}\right)\right)-h\left(\varepsilon_{n}\right)\right),
$$

for some functions $g\left(\varepsilon_{n}\right)$ and $h\left(\varepsilon_{n}\right)$ that tend to zero as $n \rightarrow \infty$ and $h\left(\varepsilon_{n}\right) \rightarrow 0$ faster than $\varepsilon_{n}$, i.e.,

$$
g\left(\varepsilon_{n}\right)=\mathrm{o}(1) \text { and } h\left(\varepsilon_{n}\right)=\mathrm{o}\left(\varepsilon_{n}\right) \text {. }
$$

With these constraints on $g\left(\varepsilon_{n}\right)$ and $h\left(\varepsilon_{n}\right)$, we see that $z_{n}$ as parametrized in (132) indeed satisfies the condition that $z_{n}=\mathrm{Q}^{-1}\left(\varepsilon_{n}(1+\mathrm{o}(1))\right)$. Now, we substitute (132) into (130) and (131) to obtain

$$
\begin{aligned}
& \left(\varepsilon_{n} \exp \left(g\left(\varepsilon_{n}\right)\right)-h\left(\varepsilon_{n}\right)\right) \\
& \times \exp \left(-\frac{S(Z) \mathrm{Q}^{-1}\left(\varepsilon_{n} \exp \left(g\left(\varepsilon_{n}\right)\right)-h\left(\varepsilon_{n}\right)\right)^{3}}{\sqrt{n}}\right) \\
& \quad+\mathrm{O}\left(\frac{1}{\sqrt{n}} \exp \left(-\frac{\mathrm{Q}^{-1}\left(\varepsilon_{n} \exp \left(g\left(\varepsilon_{n}\right)\right)-h\left(\varepsilon_{n}\right)\right)^{2}}{2}\right)\right)=\varepsilon_{n} .
\end{aligned}
$$

Solving for the function $g$ by equating coefficients in (134), we obtain

$$
\begin{aligned}
g\left(\varepsilon_{n}\right) & =\frac{S(Z) \mathrm{Q}^{-1}\left(\varepsilon_{n} \exp \left(g\left(\varepsilon_{n}\right)\right)-h\left(\varepsilon_{n}\right)\right)^{3}}{\sqrt{n}}(1+\mathrm{o}(1)) \\
& =\frac{S(Z) \mathrm{Q}^{-1}\left(\varepsilon_{n}\right)^{3}}{\sqrt{n}}(1+\mathrm{o}(1))
\end{aligned}
$$

where the last equality follows from the properties of $g$ and $h$ in (133). Solving for the function $h$ by equating coefficients in (134), we obtain

$$
\begin{array}{r}
h\left(\varepsilon_{n}\right)=\mathrm{O}\left(\frac { 1 } { \sqrt { n } } \operatorname { e x p } \left(-\frac{\mathrm{Q}^{-1}\left(\varepsilon_{n} \exp \left(g\left(\varepsilon_{n}\right)\right)-h\left(\varepsilon_{n}\right)\right)^{2}}{2}\right.\right. \\
\left.\left.+\frac{S(Z) \mathrm{Q}^{-1}\left(\varepsilon_{n} \exp \left(g\left(\varepsilon_{n}\right)\right)-h\left(\varepsilon_{n}\right)\right)^{3}}{\sqrt{n}}\right)\right) .
\end{array}
$$

Since $z_{n}=\mathrm{O}\left(n^{1 / 6}\right)$ the second term in the sum of the exponent is asymptotically negligible (compared to the first term in the exponent) and so

$$
\begin{aligned}
h\left(\varepsilon_{n}\right) & =\mathrm{O}\left(\frac{1}{\sqrt{n}} \exp \left(-\frac{\mathrm{Q}^{-1}\left(\varepsilon_{n}(1+\mathrm{o}(1))\right)^{2}}{2}\right)\right) \\
& =\mathrm{O}\left(\frac{\varepsilon_{n}}{\sqrt{n}} \mathrm{Q}^{-1}\left(\varepsilon_{n}\right)\right),
\end{aligned}
$$

where the final equality holds because $\mathrm{Q}^{-1}(s) \sim \sqrt{2 \ln \frac{1}{s}}$ as $s \rightarrow 0^{+}$; see (15). Note that both the derived $g$ and $h$ satisfy the requirements in (133). Therefore,

$$
\begin{aligned}
z_{n} & =\mathrm{Q}^{-1}\left(\varepsilon_{n} \exp \left(S(Z) \frac{\mathrm{Q}^{-1}\left(\varepsilon_{n}\right)^{3}}{\sqrt{n}}(1+\mathrm{o}(1))\right)-\mathrm{O}\left(\frac{\varepsilon_{n}}{\sqrt{n}} \mathrm{Q}^{-1}\left(\varepsilon_{n}\right)\right)\right) \\
& =\mathrm{Q}^{-1}\left(\varepsilon_{n}\left(1+\frac{S(Z) \mathrm{Q}^{-1}\left(\varepsilon_{n}\right)^{3}}{\sqrt{n}}-\mathrm{O}\left(\frac{\mathrm{Q}^{-1}\left(\varepsilon_{n}\right)}{\sqrt{n}}\right)\right)\right.
\end{aligned}
$$

where (139) follows by the fact that $\exp (x)=1+x+\mathrm{o}(x)$ as $x \rightarrow 0$. Hence, we have

$$
\begin{aligned}
\varepsilon_{n}(1 & \left.-\frac{S(Z) \Phi^{-1}\left(\varepsilon_{n}\right)^{3}}{\sqrt{n}}-\mathrm{O}\left(\frac{\Phi^{-1}\left(\varepsilon_{n}\right)}{\sqrt{n}}\right)\right)=\mathrm{Q}\left(z_{n}\right) \\
= & \Phi\left(-z_{n}\right)=\Phi\left(\zeta_{n}\left(1-\varepsilon_{n}\right)\right)
\end{aligned}
$$

completing the proof of (73).

\section{APPENDIX C}

\section{PROOF OF LEMMA 8}

After some algebra, we get

$$
\mu\left\{\sum_{i=1}^{n} f_{i} \geq a\right\}=\mathrm{e}^{-n \Lambda_{\mu_{f}}^{*}(a)} \int_{0}^{\infty} \mathrm{e}^{-s t \sqrt{n \Lambda_{\mu_{f}^{\prime \prime}}(s)}} \mathrm{d} F_{n}(t),
$$

where $F_{n}$ is a distribution function of the r.v. $W_{n}$ given by

$$
W_{n}=\frac{1}{\sqrt{n \Lambda_{\mu_{f}}^{\prime \prime}(s)}} \sum_{i=1}^{n}\left(U_{i}-a\right),
$$

and $U_{1}, \ldots, U_{n}$ are i.i.d. r.v.'s with generic distribution $\tilde{\mu}_{f}$ constructed by the Radon-Nikodym derivative

$$
\frac{\mathrm{d} \tilde{\mu}_{f}}{\mathrm{~d} \mu_{f}}(t)=\mathrm{e}^{s t-\Lambda_{\mu_{f}}(s)} .
$$

Then, Lemma 8 can be proven by applying the Edgeworth expansion (cf. [11, Chapter XVI.4] or [12, Chapter VI.3]) to the distribution $F_{n}$ in (141); see the proof of [13, Theorem 3.7.4].

\section{APPENDIX D}

PROOF OF LEMMA 9

For the sake of brevity, we write

$$
\alpha_{n}=\alpha_{n}(t):=a n+a_{n}(t) .
$$

As in [13, Exercise 2.2.24], we observe that $\Lambda_{\mu_{f}}^{*}(\tilde{a})$ is of class $C^{\infty}$ in $\tilde{a} \in \operatorname{int}\left(\mathcal{F}_{\mu_{f}}\right)$, where $\mathcal{F}_{\mu_{f}}:=\left\{\Lambda_{\mu_{f}}^{\prime}(\tilde{s}) \mid \tilde{s} \in \operatorname{int}\left(\mathcal{D}_{\mu_{f}}\right)\right\}$. Thus, since $s \in \operatorname{int}\left(\mathcal{D}_{\mu_{f}}\right)$, it follows from (77), (81), and (144) that $\alpha_{n} / n \in \operatorname{int}\left(\mathcal{F}_{\mu_{f}}\right)$ for sufficiently large $n$. Henceforth, we 
assume that $n$ is large enough. Noting this fact, denote by $s_{n}=s_{n}(t)$ the root of the equation $n \Lambda_{\mu_{f}}^{\prime}(\tilde{s})=\alpha_{n}$ with respect to $\tilde{s} \in \operatorname{int}\left(\mathcal{D}_{\mu_{f}}\right)$.

Since $\Lambda_{\mu_{f}}^{\prime}(\cdot)$ is of class $C^{\infty}$ on $\operatorname{int}\left(\mathcal{D}_{\mu_{f}}\right)$, and since (81) implies that $\alpha_{n} \rightarrow a$ uniformly on $\mathcal{I}_{n}$ as $n \rightarrow \infty$, we see that $s_{n} \rightarrow s$ as $n \rightarrow \infty$ uniformly on $\mathcal{I}_{n}$. In addition, it follows from Taylor's theorem for $v \mapsto \Lambda_{\mu_{f}}^{\prime}(v)$ around $v=s$ that

$$
\frac{\alpha_{n}}{n}=\Lambda_{\mu_{f}}^{\prime}\left(s_{n}\right)=a+\Lambda_{\mu_{f}}^{\prime \prime}(s)\left(s_{n}-s\right)+\mathrm{O}\left(\left(s_{n}-s\right)^{2}\right)
$$

uniformly on $\mathcal{I}_{n}$ as $n \rightarrow \infty$, which is equivalent to

$$
s_{n}=s+\frac{a_{n}(t)}{n \Lambda_{\mu_{f}}^{\prime \prime}(s)}+\mathrm{O}\left(\left(s_{n}-s\right)^{2}\right)
$$

uniformly on $\mathcal{I}_{n}$ as $n \rightarrow \infty$. Therefore, since $s_{n} \rightarrow s$ as $n \rightarrow \infty$ uniformly in $t \in \mathcal{I}_{n}$, we observe that

$$
s_{n}-s=\left(\frac{a_{n}(t)}{n \Lambda_{\mu_{f}}^{\prime \prime}(s)}\right)(1+o(1))
$$

uniformly on $\mathcal{I}_{n}$ as $n \rightarrow \infty$. On the other hand, it follows from (77), (146), and Taylor's theorem for $v \mapsto \Lambda_{\mu_{f}}(v)$ around $v=s$ that there exist real sequences $r_{f}^{(1)}(n, t)=\mathrm{o}(1)$ and $r_{f}^{(2)}(n, t)=\mathrm{O}(1)$ uniformly on $\mathcal{I}_{n}$ as $n \rightarrow \infty$ such that (148) (at the top of the next page) holds. This is equivalent to

$$
\Lambda_{\mu_{f}}^{*}\left(\frac{\alpha_{n}}{n}\right)=K_{f}(n, t),
$$

where $K_{f}(n, t)$ is defined as

$$
\begin{aligned}
K_{f}(n, s, t) \\
:=n \Lambda_{\mu_{f}}^{*}(a)+s a_{n}(t) \\
+\frac{a_{n}(t)^{2}}{2 n \Lambda_{\mu_{f}}^{\prime \prime}(s)}\left(1+r_{f}^{(1)}(n, t)\left(2+r_{f}^{(1)}(n, t)\right)\right. \\
\left.\quad+r_{f}^{(2)}(n, t)\left(1+r_{f}^{(1)}(n, t)\right)^{3} \frac{2 a_{n}(t)}{n \Lambda_{\mu_{f}^{\prime \prime}}^{\prime \prime}(s)^{2}}\right) .
\end{aligned}
$$

Finally, it follows from Taylor's theorem for $v \mapsto \Lambda_{\mu_{f}}^{\prime \prime}(v)$ around $v=s$ that there exists a real sequence $r_{f}^{(3)}(n, t)=\mathrm{O}(1)$ uniformly on $\mathcal{I}_{n}$ as $n \rightarrow \infty$ such that

$$
\begin{aligned}
\Lambda_{\mu_{f}}^{\prime \prime}\left(s_{n}\right) & =\Lambda_{\mu_{f}}^{\prime \prime}(s)+\Lambda_{\mu_{f}}^{\prime \prime \prime}(s)\left(s_{n}-s\right)+r_{f}^{(3)}(n, t)\left(s_{n}-s\right)^{2} \\
& =\Lambda_{\mu_{f}}^{\prime \prime}(s)\left(1+R_{f}(n, t)\right)
\end{aligned}
$$

where $R_{f}(n, t)$ is defined as

$$
\begin{aligned}
R_{f}(n, t):= & \frac{\Lambda_{\mu_{f}}^{\prime \prime \prime}(s)\left(1+r_{f}^{(1)}(n, t)\right)}{\Lambda_{\mu_{f}}^{\prime \prime}(s)} \frac{a_{n}(t)}{n \Lambda_{\mu_{f}}^{\prime \prime}(s)} \\
& +\frac{r_{f}^{(3)}(n, t)\left(1+r_{f}^{(1)}(n, t)\right)^{2}}{\Lambda_{\mu_{f}}^{\prime \prime}(s)}\left(\frac{a_{n}(t)}{n \Lambda_{\mu_{f}}^{\prime \prime}(s)}\right)^{2} .
\end{aligned}
$$

By (81), we readily see that $R_{f}(n, t)=\mathrm{o}(1)$ uniformly on $\mathcal{I}_{n}$ as $n \rightarrow \infty$. Applying the above asymptotic results to Lemma 8 , we obtain Lemma 9, as desired.

\section{ACKNOWLEDGEMENT}

The authors are also grateful to the Associate Editor Prof. Tobias Koch and the reviewers for their valuable and detailed comments.

\section{REFERENCES}

[1] T. S. Han, "Weak variable-length source coding," IEEE Trans. Inf. Theory, vol. 46, no. 4, pp. 1217-1226, Jul. 2000.

[2] H. Koga and H. Yamamoto, "Asymptotic properties on codeword lengths of an optimal fv code for general sources," IEEE Trans. Inf. Theory, vol. 51, no. 4, pp. 1546-1555, Apr. 2005.

[3] J. Wolfowitz, Coding Theorems of Information Theory, 3rd ed. New York: Springer-Verlag, 1978.

[4] A. D. Wyner, "An upper bound on the entropy series," Inf. Control, vol. 20, no. 2, pp. 176-181, Mar. 1972.

[5] N. Alon and A. Orlitsky, "A lower bound on the expected length of oneto-one codes," IEEE Trans. Inf. Theory, vol. 40, no. 5, pp. 1670-1672, Sept. 1994.

[6] W. Szpankowski, "A one-to-one code and its anti-redundancy," IEEE Trans. Inf. Theory, vol. 54, no. 10, pp. 4762-4766, Oct. 2008.

[7] W. Szpankowski and S. Verdú, "Minimum expected length of fixed-tovariable lossless compression without prefix constraints," IEEE Trans. Inf. Theory, vol. 57, no. 7, pp. 4017-4025, Jul. 2011.

[8] V. Kostina, Y. Polyanskiy, and S. Verdú, "Variable-length compression allowing errors," IEEE Trans. Inf. Theory, vol. 61, no. 8, pp. 4316-4330, Aug. 2015.

[9] I. Kontoyiannis and S. Verdú, "Optimal lossless data compression: Nonasymptotics and asymptotics," IEEE Trans. Inf. Theory, vol. 60, no. 2 , pp. 777-795, Feb. 2014.

[10] S. Verdú, "Teaching IT," in XXVIII Shannon Lecture at IEEE Int. Symp. Inf. Theory, Nice, France, Jun. 2007.

[11] W. Feller, An Introduction to Probability Theory and Its Applications, 2nd ed. New York: Wiley, 1971, vol. 2.

[12] V. V. Petrov, Sums of Independent Random Variables. Berlin, Germany: Springer-Verlag, 1975.

[13] A. Dembo and O. Zeitouni, Large Deviations Techniques and Applications, 2nd ed. New York: Springer-Verlag, 1998.

[14] V. Strassen, "Asymptotische Abschätzungen in Shannon's Informationstheorie," in Trans. 3rd Prague Conf. Inf. Theory. Prague: Academia, 1962, pp. 689-723.

[15] M. Hayashi, "Second-order asymptotics in fixed-length source coding and intrinsic randomness," IEEE Trans. Inf. Theory, vol. 54, no. 10, pp. 4619-4637, Oct. 2008.

[16] — - "Information spectrum approach to second-order coding rate in channel coding," IEEE Trans. Inf. Theory, vol. 55, no. 11, pp. 49474966, Nov. 2009.

[17] Y. Polyanskiy, H. V. Poor, and S. Verdú, "Channel coding rate in the finite blocklength regime," IEEE Trans. Inf. Theory, vol. 56, no. 5, pp. 2307-2359, May 2010.

[18] V. Y. F. Tan, "Asymptotic estimates in information theory with nonvanishing error probabilities," Found. Trends Commun. Inf. Theory, vol. 11, no. 1-2, pp. 1-184, 2014.

[19] P. Moulin, "The log-volume of optimal codes for memoryless channels, asymptotically within a few nats," IEEE Trans. Inf. Theory, vol. 63, no. 4, pp. 2278-2313, Apr. 2017.

[20] M. Hayashi, "Semi-finite length analysis for information theoretic tasks," Nov. 2018, [Online]. Available at https://arxiv.org/abs/1811.00262v2 (this study was presented in part at IEEE Int. Symp. Inf. Theory, Paris, France, Jul. 2019, pp. 952-956)

[21] Y. Altuğ and A. B. Wagner, "Moderate deviations in channel coding," IEEE Trans. Inf. Theory, vol. 60, no. 8, pp. 4417-4426, Aug. 2014.

[22] —_ "Refinement of the sphere-packing bound: asymmetric channels," IEEE Trans. Inf. Theory, vol. 60, no. 3, pp. 1592-1614, May 2014.

[23] — , "Refinement of the random coding bound," IEEE Trans. Inf. Theory, vol. 60, no. 10, pp. 6005-6023, Oct. 2014.

[24] J. Honda, "Exact asymptotics for the random coding error probability," in Proc. IEEE Int. Symp. Inf. Theory, Hong Kong, Jun. 2015, pp. 91-95.

[25] — , "Exact asymptotics of random coding error probability for general memoryless channels," in Proc. IEEE Int. Symp. Inf. Theory, Vail, CO, USA, Jun. 2018, pp. 1844-1848.

[26] Y. Altuğ and A. B. Wagner, "On exact asymptotics of the error probability in channel coding: symmetric channels," IEEE Trans. Inf. Theory, vol. 67, no. 2, pp. 844-868, Feb. 2021.

[27] N. Merhav, "Universal coding with minimum probability of codeword length overflow," IEEE Trans. Inf. Theory, vol. 37, no. 3, pp. 556-563, May 1991.

[28] O. Kosut and L. Sankar, "Asymptotics and non-asymptotics for universal fixed-to-variable source coding," IEEE Trans. Inf. Theory, vol. 63, no. 6, pp. 3753-3772, Jun. 2017. 


$$
\begin{aligned}
\frac{\alpha_{n} s_{n}}{n}-\Lambda_{\mu_{f}}^{*}\left(\frac{\alpha_{n}}{n}\right)= & \Lambda_{\mu_{f}}\left(s_{n}\right) \\
= & \Lambda_{\mu_{f}}(s)+\Lambda_{\mu_{f}}^{\prime}(s)\left(s_{n}-s\right)+\frac{\Lambda_{\mu_{f}}^{\prime \prime}(s)}{2}\left(s_{n}-s\right)^{2}+r_{f}^{(1)}(n, t)\left(s_{n}-s\right)^{3} \\
= & a s-\Lambda_{\mu_{f}}^{*}(a)+a\left(s_{n}-s\right)+\frac{\Lambda_{\mu_{f}}^{\prime \prime}(s)}{2}\left(s_{n}-s\right)^{2}+r_{f}^{(1)}(n, t)\left(s_{n}-s\right)^{3} \\
= & \left(a-\frac{\alpha_{n}}{n}\right) s-\Lambda_{\mu_{f}}^{*}(a)+\frac{\alpha_{n} s_{n}}{n}+\left(a-\frac{\alpha_{n}}{n}\right)\left(s_{n}-s\right)+\frac{\Lambda_{\mu_{f}}^{\prime \prime}(s)}{2}\left(s_{n}-s\right)^{2}+r_{f}^{(1)}(n, t)\left(s_{n}-s\right)^{3} \\
= & \left(a-\frac{\alpha_{n}}{n}\right) s-\Lambda_{\mu_{f}}^{*}(a)+\frac{\alpha_{n} s_{n}}{n}-\frac{a_{n}(t)^{2}}{n^{2} \Lambda_{\mu_{f}}^{\prime \prime}(s)} \\
& \quad+\left(\frac{a_{n}(t)^{2}}{2 n^{2} \Lambda_{\mu_{f}}^{\prime \prime}(s)}\right)\left(1+r_{f}^{(1)}(n, t)\right)^{2}+r_{f}^{(2)}(n, t)\left(\frac{a_{n}(t)}{n \Lambda_{\mu_{f}}^{\prime \prime}(s)}\right)^{3}\left(1+r_{f}^{(1)}(n, t)\right)^{3} \\
= & \left(a-\frac{\alpha_{n}}{n}\right) s-\Lambda_{\mu_{f}}^{*}(a)+\frac{\alpha_{n} s_{n}}{n}-\frac{a_{n}(t)^{2}}{2 n^{2} \Lambda_{\mu_{f}}^{\prime \prime}(s)} \\
& \quad+\left(\frac{a_{n}(t)^{2}}{2 n^{2} \Lambda_{\mu_{f}}^{\prime \prime}(s)}\right)\left(2 r_{f}^{(1)}(n, t)+r_{f}^{(1)}(n, t)^{2}\right)+r_{f}^{(2)}(n, t)\left(\frac{a_{n}(t)}{n \Lambda_{\mu_{f}}^{\prime \prime}(s)}\right)^{3}\left(1+r_{f}^{(1)}(n, t)\right)^{3} .
\end{aligned}
$$

[29] N. Iri and O. Kosut, "Fine asymptotics for universal one-to-one compression of parametric sources," IEEE Trans. Inf. Theory, vol. 65, no. 4, pp. 2442-2458, Apr. 2019.

[30] R. Nomura and H. Yagi, "Optimum overflow thresholds in variablelength source coding allowing non-vanishing error probability," IEEE Trans. Inf. Theory, vol. 65, no. 12, pp. 8213-8221, Dec. 2019.

[31] M. Kovačević, I. Stanojević, and V. Šenk, "Some properties of Rényi entropy over countably infinite alphabets," Probl. Inf. Transm., vol. 49 , no. 2, pp. 99-110, 2013.

[32] I. Csiszár and J. Körner, Information Theory: Coding Theorems for Discrete Memoryless Systems, 2nd ed. Cambridge, UK: Cambridge University Press, 2011.

[33] J. M. Blair, C. A. Edwards, and J. H. Johnson, "Rational Chebyshev approximations for the inverse of the error function," Math. Comput., vol. 30, no. 136, pp. 827-830, Oct. 1976.

[34] Y. Sakai and V. Y. F. Tan, "Variable-length source dispersions differ under maximum and average error criteria," IEEE Trans. Inf. Theory, vol. 66 , no. 12 , pp. $7565-7587$, Oct. 2020

[35] W. Szpankowski, Average Case Analysis of Algorithms in Sequences. New York: Wiley, 2000.

[36] S. Saito and T. Matsushima, "Evaluation of overflow probability of Bayes code in moderate deviation regime," IEICE Trans. Fundamentals, vol. E100-A, no. 12, pp. 2728-2731, Dec. 2017.

[37] J. L. Massey, "Guessing and entropy," in Proc. IEEE Int. Symp. Inf. Theory, Chicago, IL, USA, June 1994, p. 204.

[38] E. Arıkan, "An inequality on guessing and its application to sequential decoding," IEEE Trans. Inf. Theory, vol. 42, no. 1, pp. 99-105, Jan. 1996.

[39] S. Kuzuoka, "On the conditional smooth Rényi entropy and its applications in guessing and source coding," IEEE Trans. Inf. Theory, vol. 66 , no. 3, pp. 1674-1690, Mar. 2020.

[40] V. Y. F. Tan and M. Hayashi, "Analysis of remaining uncertainties and exponents under various conditional Rényi entropies," IEEE Trans. Inf. Theory, vol. 64, no. 5, pp. 3734-3755, Jan. 2018.

[41] V. Y. F. Tan and M. Tomamichel, "The third-order term in the normal approximation for the AWGN channel," IEEE Trans. Inf. Theory, vol. 61, no. 5, pp. 2430-2438, May 2015

[42] M. Tomamichel and V. Y. F. Tan, "A tight upper bound for the thirdorder asymptotics for most discrete memoryless channels," IEEE Trans. Inf. Theory, vol. 59, no. 12, pp. 7041-7051, Dec. 2013.

Yuta Sakai (Member, IEEE) was born in Japan in 1992. He is currently an Assistant Professor at the Department of Electronics and Computer Science, Graduate School of Engineering, University of Hyogo. Her was formerly a
Research Fellow in the Department of Electrical and Computer Engineering at the National University of Singapore (NUS) from 2018-2020. He received the B.E. and M.E. degrees in the Department of Information Science from the University of Fukui in 2014 and 2016, respectively, and the Ph.D. degree in the Advanced Interdisciplinary Science and Technology from the University of Fukui in 2018. His research interests include information theory and coding theory.

Recep Can Yavas (S'19) is currently a Ph.D. candidate in electrical engineering at the California Institute of Technology (Caltech). He received the B.S. degree from Bilkent University in Ankara, Turkey, in 2016 and the M.S. degree from Caltech in 2017, both in electrical engineering. His research interests include information theory and probability theory.

Vincent Y. F. Tan (S'07-M'11-SM'15) was born in Singapore in 1981. He received the B.A. and M.Eng. degrees in electrical and information science from Cambridge University in 2005, and the Ph.D. degree in electrical engineering and computer science (EECS) from the Massachusetts Institute of Technology (MIT) in 2011. He is currently a Dean's Chair Associate Professor with the Department of Electrical and Computer Engineering and the Department of Mathematics, National University of Singapore (NUS). His research interests include information theory, machine learning, and statistical signal processing.

Dr. Tan is a member of the IEEE Information Theory Society Board of Governors. He was an IEEE Information Theory Society Distinguished Lecturer from 2018 to 2019. He received the MIT EECS Jin-Au Kong Outstanding Doctoral Thesis Prize in 2011, the NUS Young Investigator Award in 2014, the Singapore National Research Foundation (NRF) Fellowship (Class of 2018), and the NUS Young Researcher Award in 2019. He is currently serving as an Associate Editor for the IEEE Transactions ON Signal Processing and for the IEEE TRANSACTIONS ON INFORMATION THEORY. 